\title{
FERMILAB-SLIDES-21-041-PPD
}

This manuscript has been authored by Fermi Research Alliance, LLC under Contract No. DE-AC02-07CH11359

with the U.S. Department of Energy, Office of Science, Office of High Energy Physics

\section{SUSY at the LHC}

Anadi Canepa (acanepa @ fnal.gov) PPC2021: XIV International Workshop on Interconnections between Particle Physics and Cosmology 17-21 May 2021, Univ. of Oklahoma
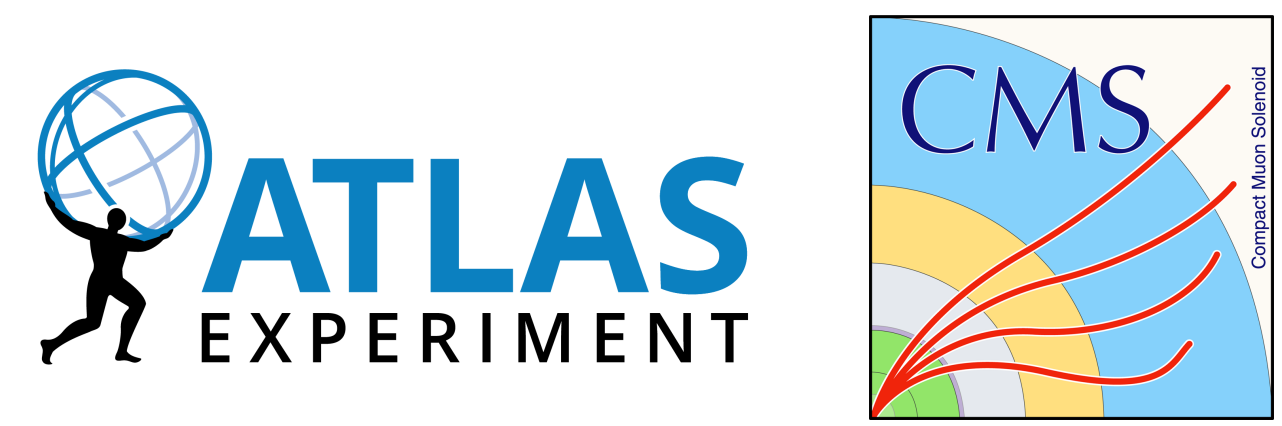


\section{Outline}

- Introduction to SUSY

- ATLAS and CMS at Run 2

- Latest results from SUSY searches

- Outlook
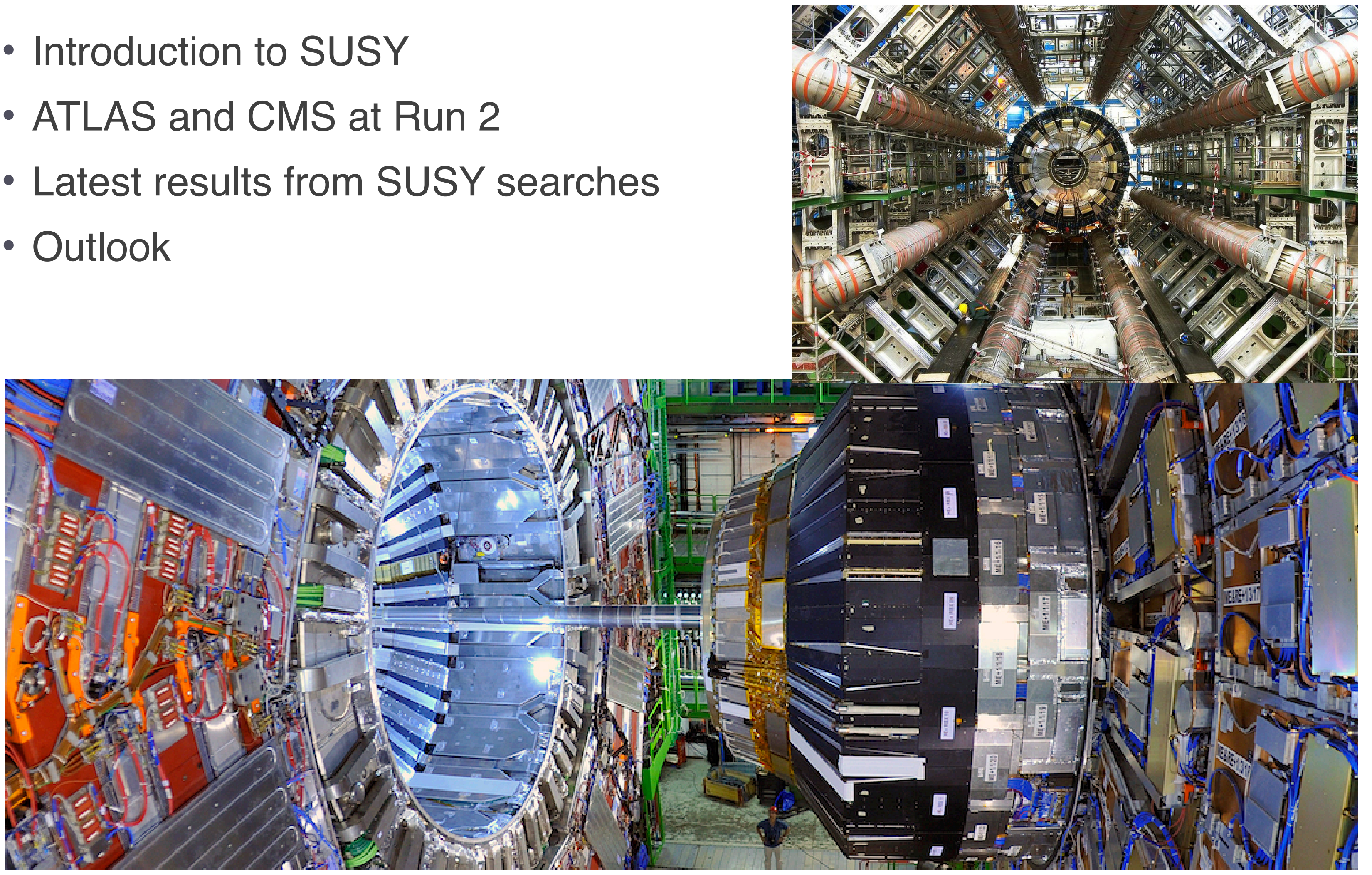


\section{A brief reminder of SUSY}

- The introduction of a new space-time symmetry requires the existence of SUSY partners of the SM particles with predictable couplings

- The experiments at LHC (LEP, Tevatron!) have adopted well motivated guiding principles to cast a wide net to search for SUSY

- R-parity conservation (pair production, stable lightest SUSY particle)
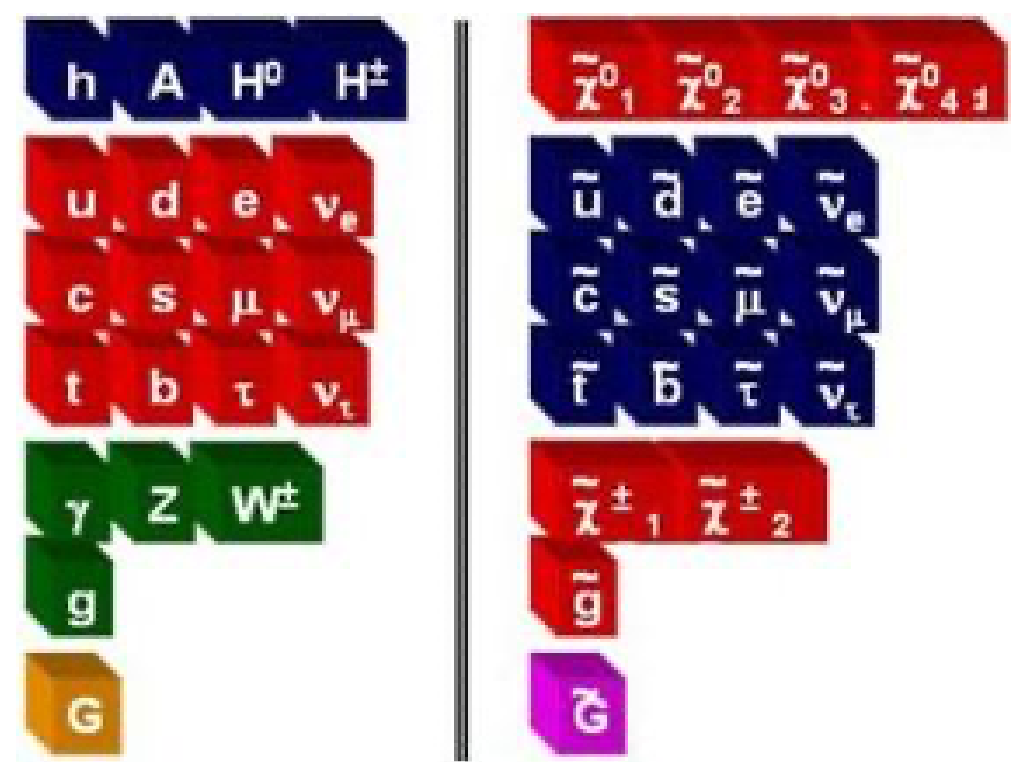

- Naturalness (gluinos, stops, electro-weakinos at TeV scale)
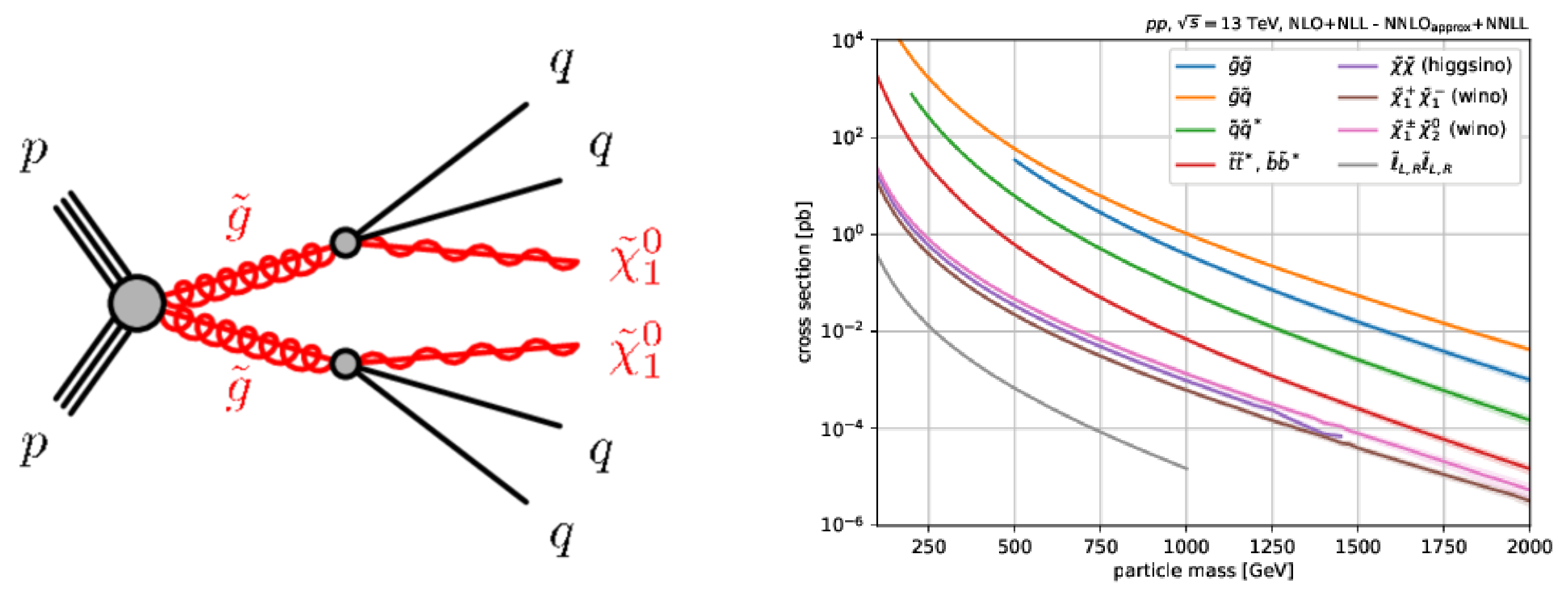


\section{"Classic" SUSY search}

- Events with large ETmiss and energetic jets

- SRs based on observables proxies of the SUSY mass scale and topological properties

- Control and validation regions for background estimate

- Simultaneous fit in both CRs and SRs (up to hundreds!) to look for excess

- Interpretation of results in simplified models
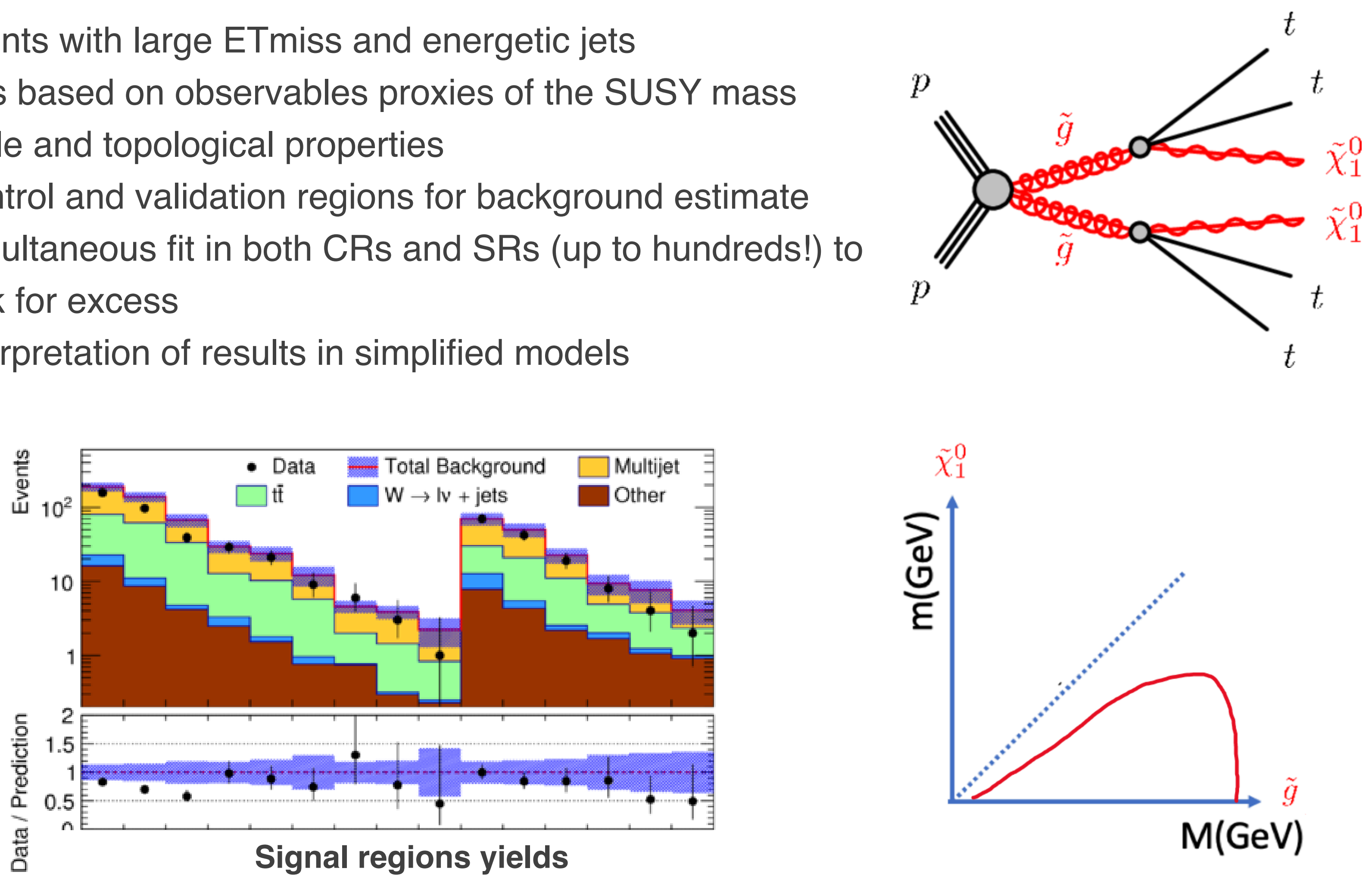


\section{Where is SUSY?}

- No significant deviations from SM expectations

- SUSY likely hidden in challenging regions of parameter space

- Higher mass scale

- Identify very high pT objects

- Compressed spectra

- Identify very low pT objects

- Long-lived

- Paradigm shift in object reconstruction

- R-parity violation / Stealth

- Explore events with low ETmiss

- Unexpected

Focus on novel techniques targeting challenging signatures

All results from ATLAS and CMS searches at: https://twiki.cern.ch/twiki/bin/view/AtlasPublic/SupersymmetryPublicResults https://twiki.cern.ch/twiki/bin/view/CMSPublic/PhysicsResultssus
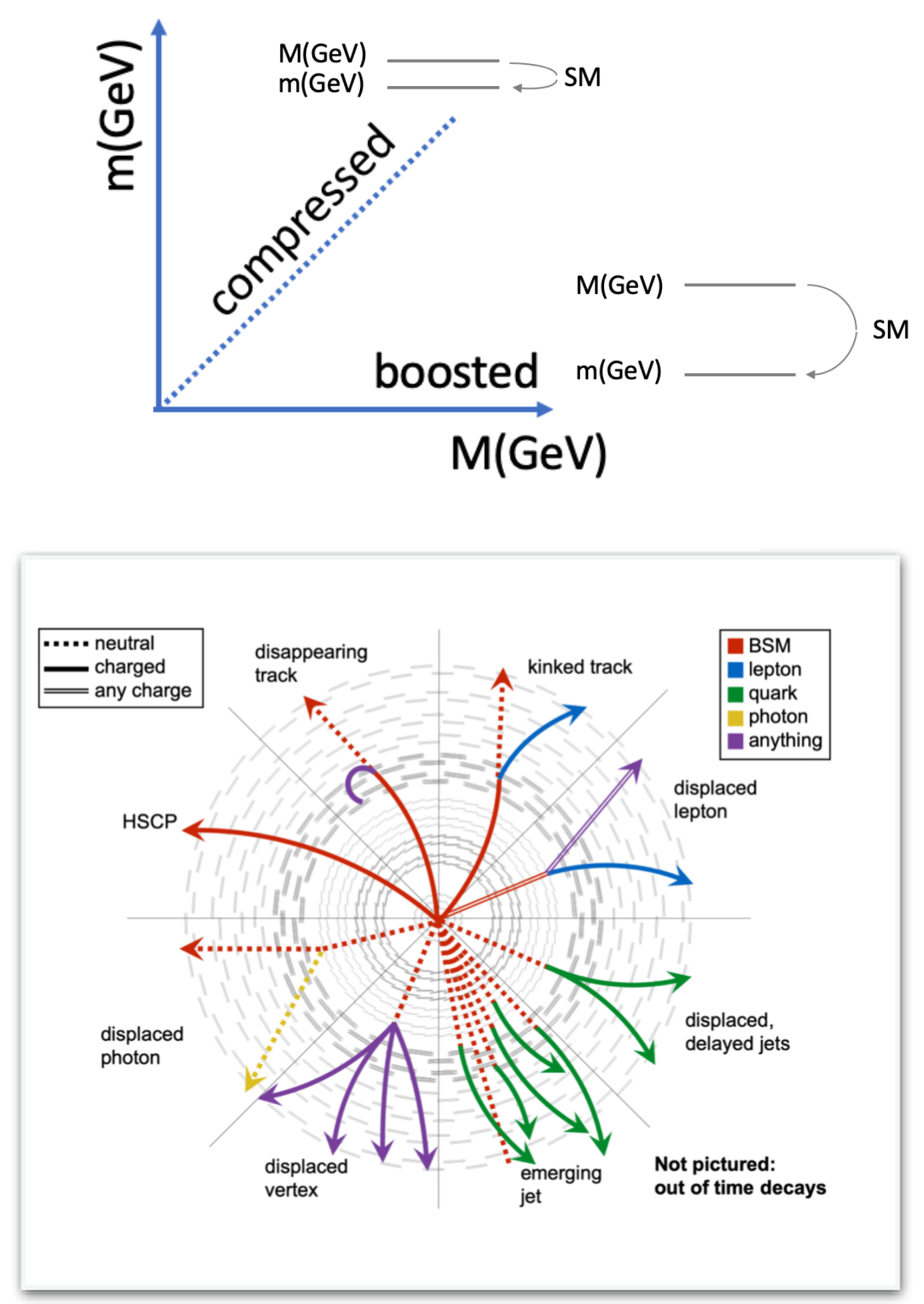


\section{The LHC Run 2}
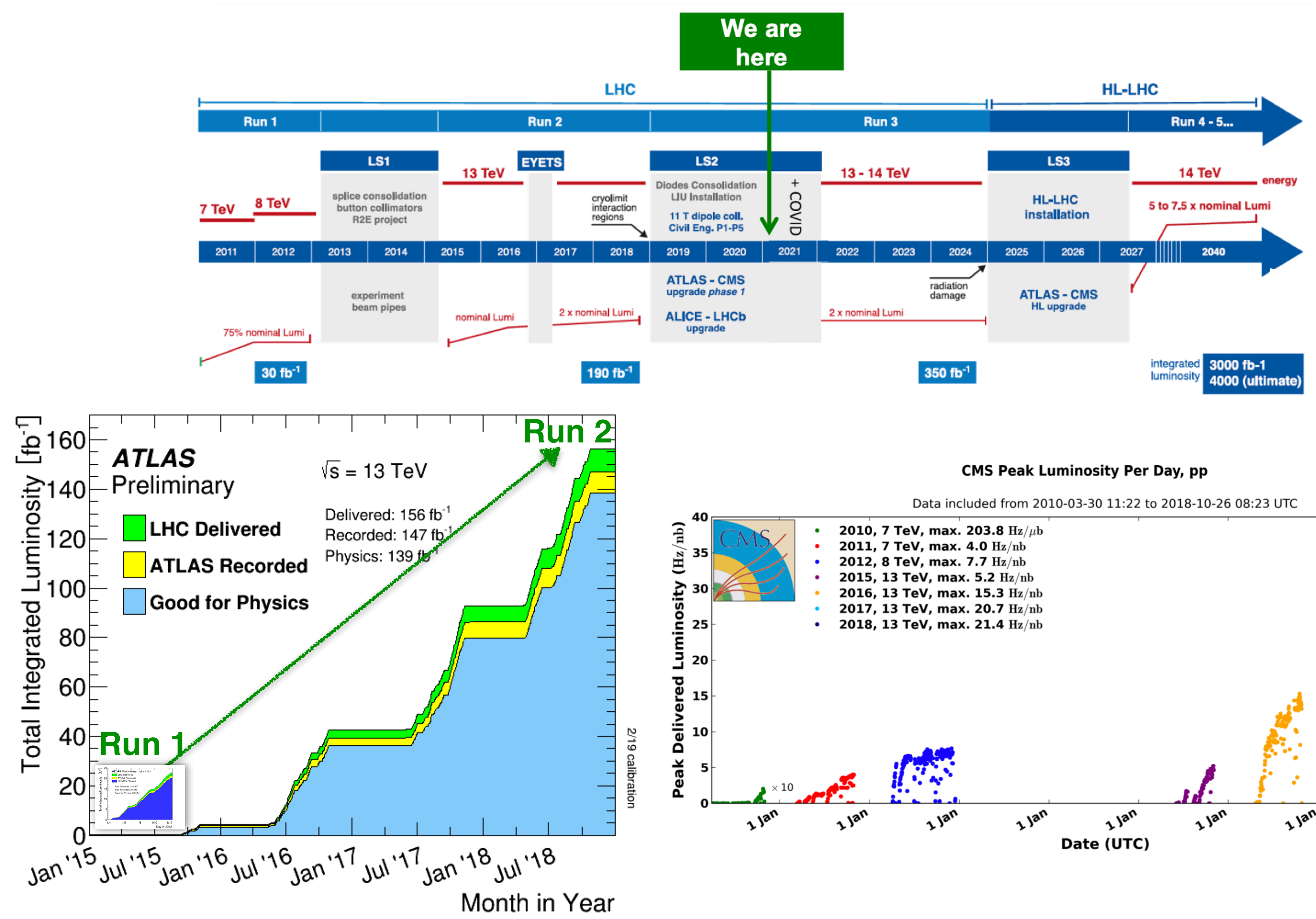

CMS Peak Luminosity Per Day, pp

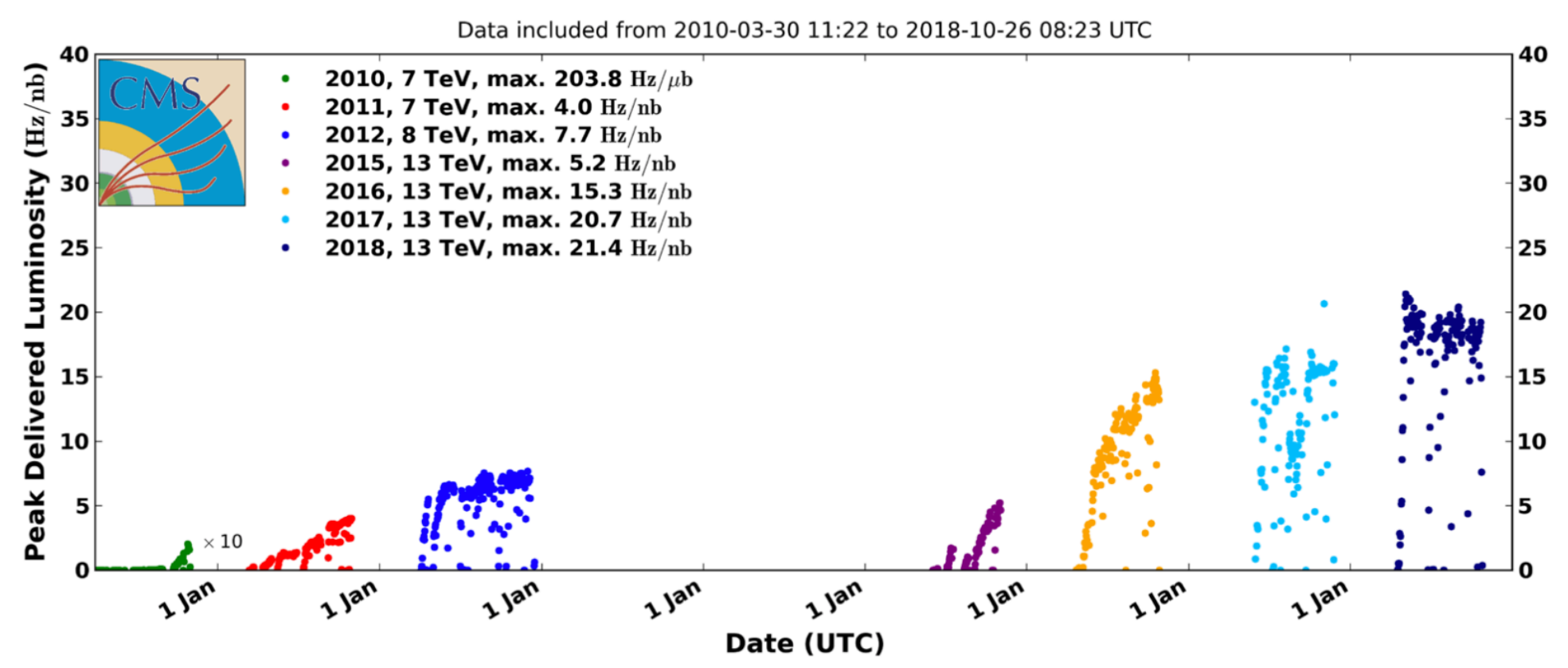




\section{Run 2: Unprecedented dataset for HEP}

- Revolution in data reconstruction and analysis thanks to modern machine learning algorithms

- Moving from MultiVariate to Deep Neural Networks

- Ability to extract information from highly complex data sets

- Leap forward in reconstructing very challenging signatures from boosted decays of top quarks and bosons using substructures, soft-drop mass, heavy flavor information, and more

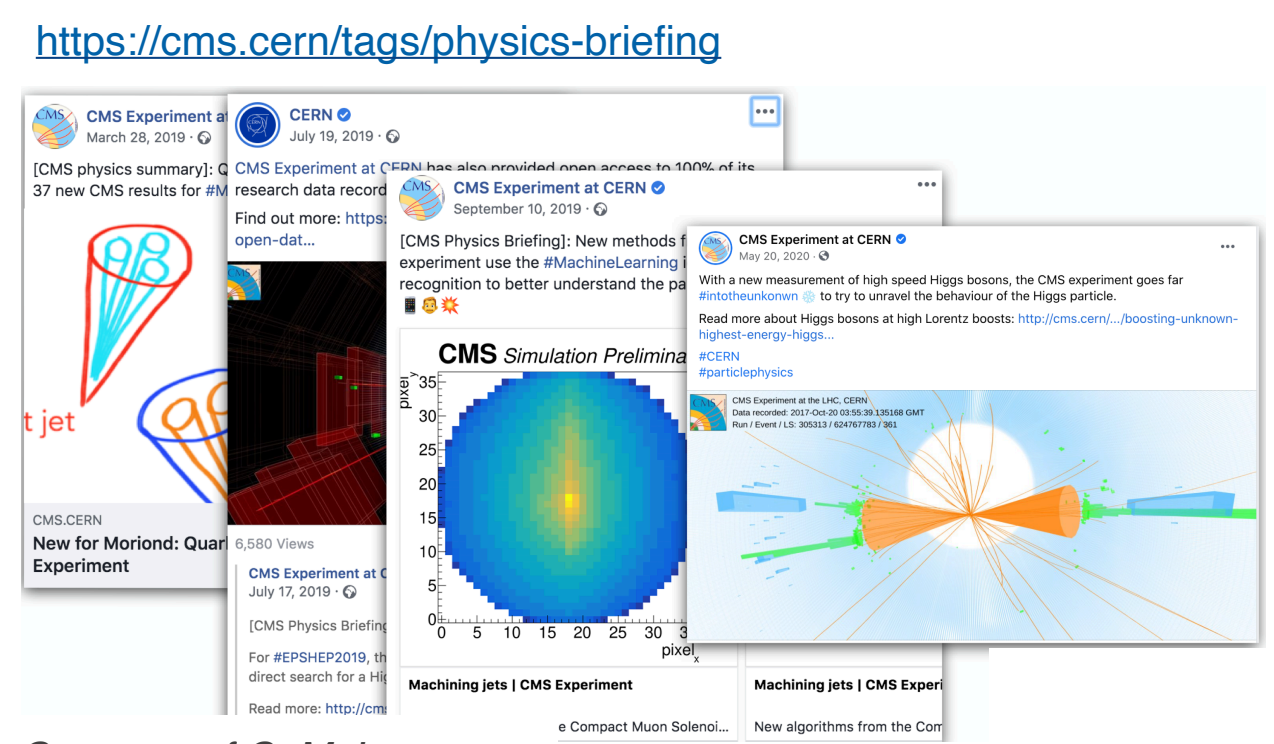

Courtesy of C. McLean
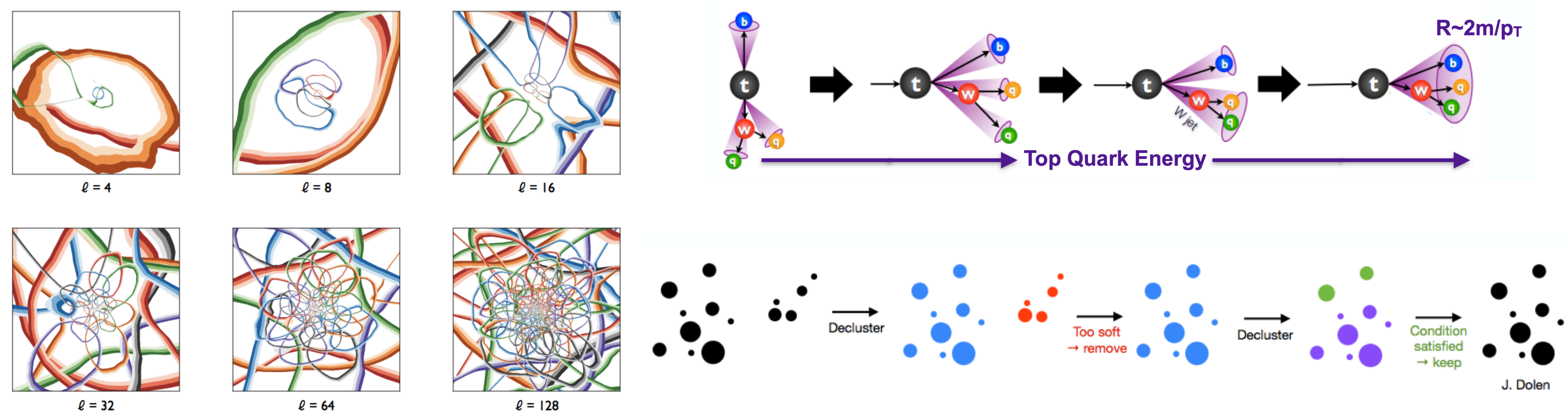

J. Dolen 


\section{Searches for Gluinos and Squarks}




\section{"Classic" searches for gluinos and LF-squarks}

- Legacy search for strongly produced light-flavor squarks and gluinos in the final state with one lepton, jets, and ETmiss

- Large Run 2 data enables classification of events in several SRs, CRs and excellent control of systematic uncertainties on tails of background

- e.g. jet energy scale few \%
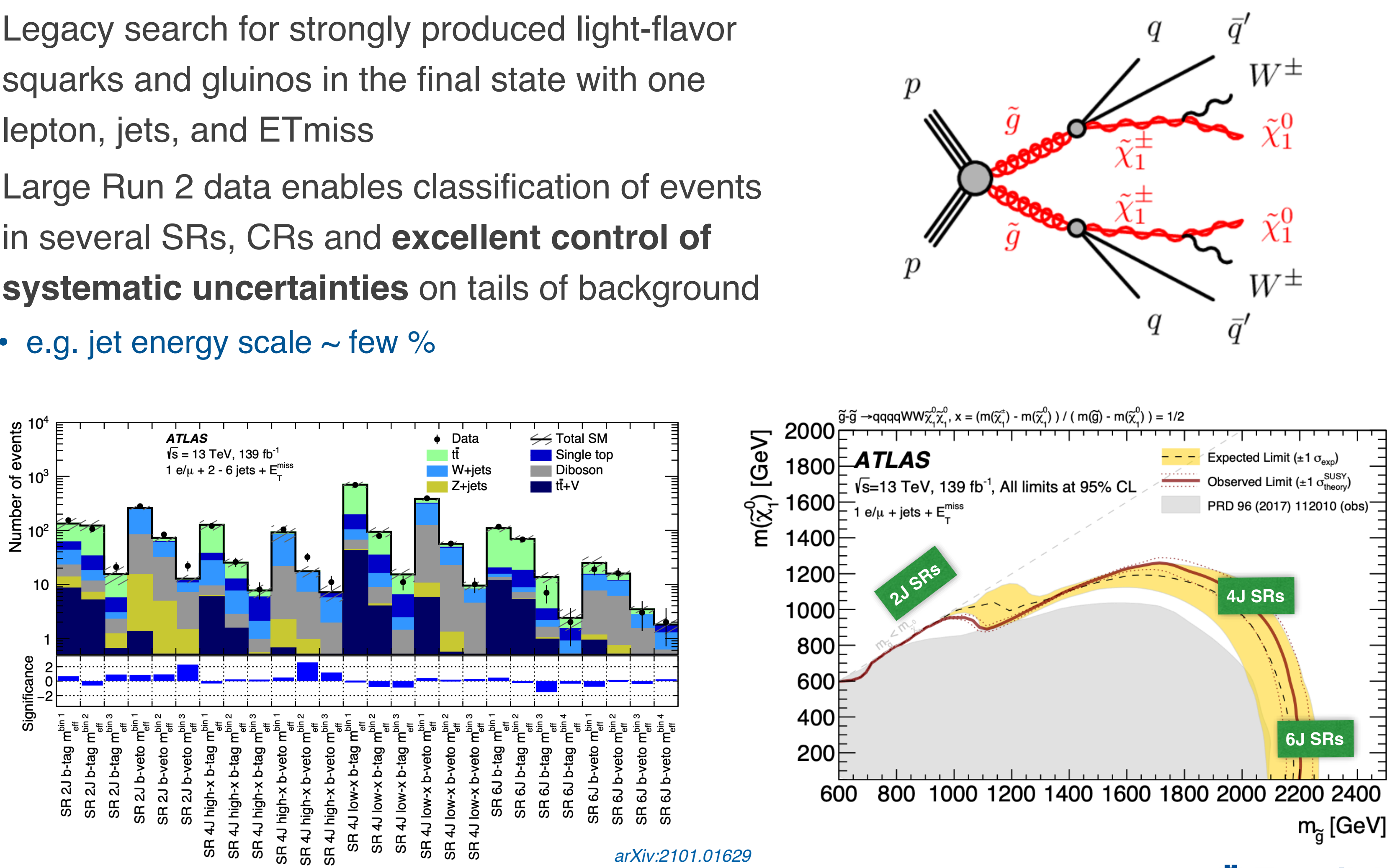


\section{Searches for Stops and Sbottoms}




\section{Searches for stops directly produced and in gluino decays}

- Search in events with multiple (b-)jets, no leptons, and large ETmiss

- Advanced classification of events in 183 SRs

- Novel techniques based on Deep Neural Networks to reconstruct highly boosted top quarks expected from high mass stop signals

- Soft b-tagging (pT < 20GeV) optimized for compressed spectra
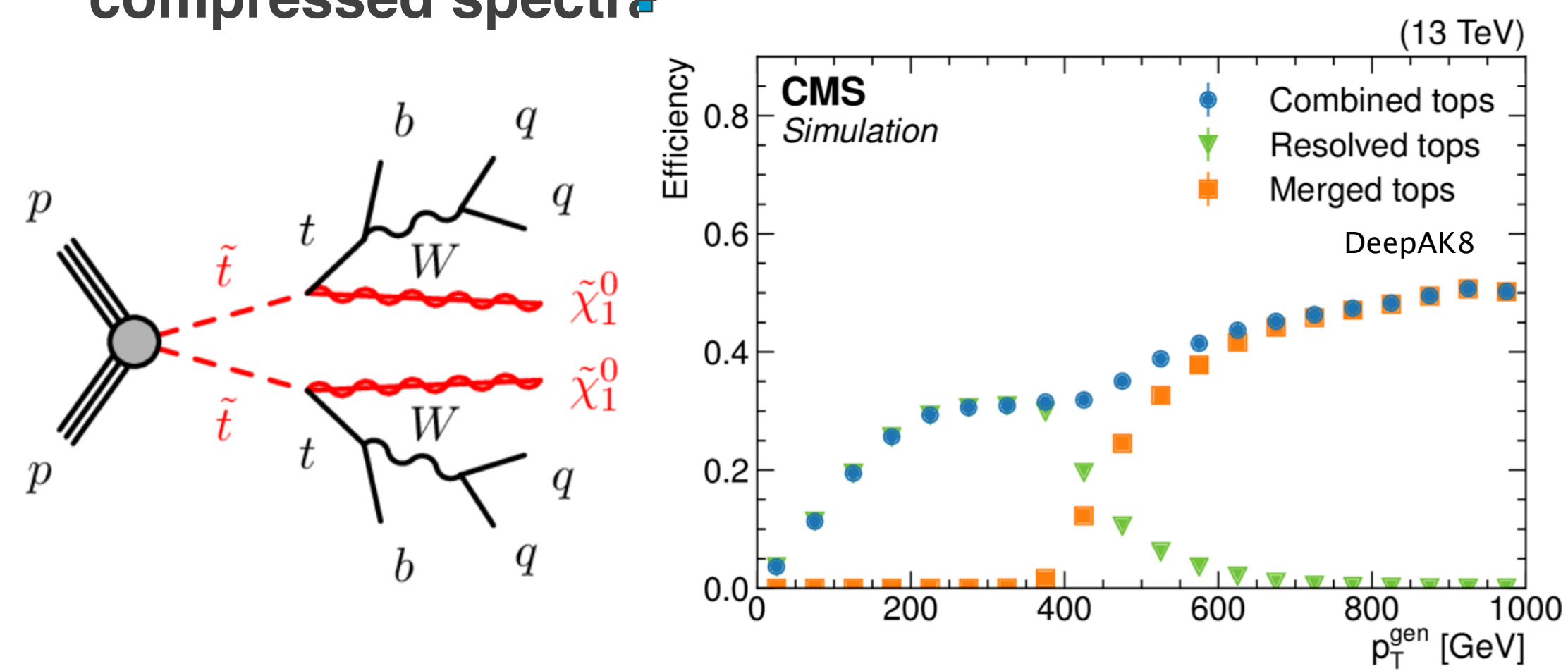
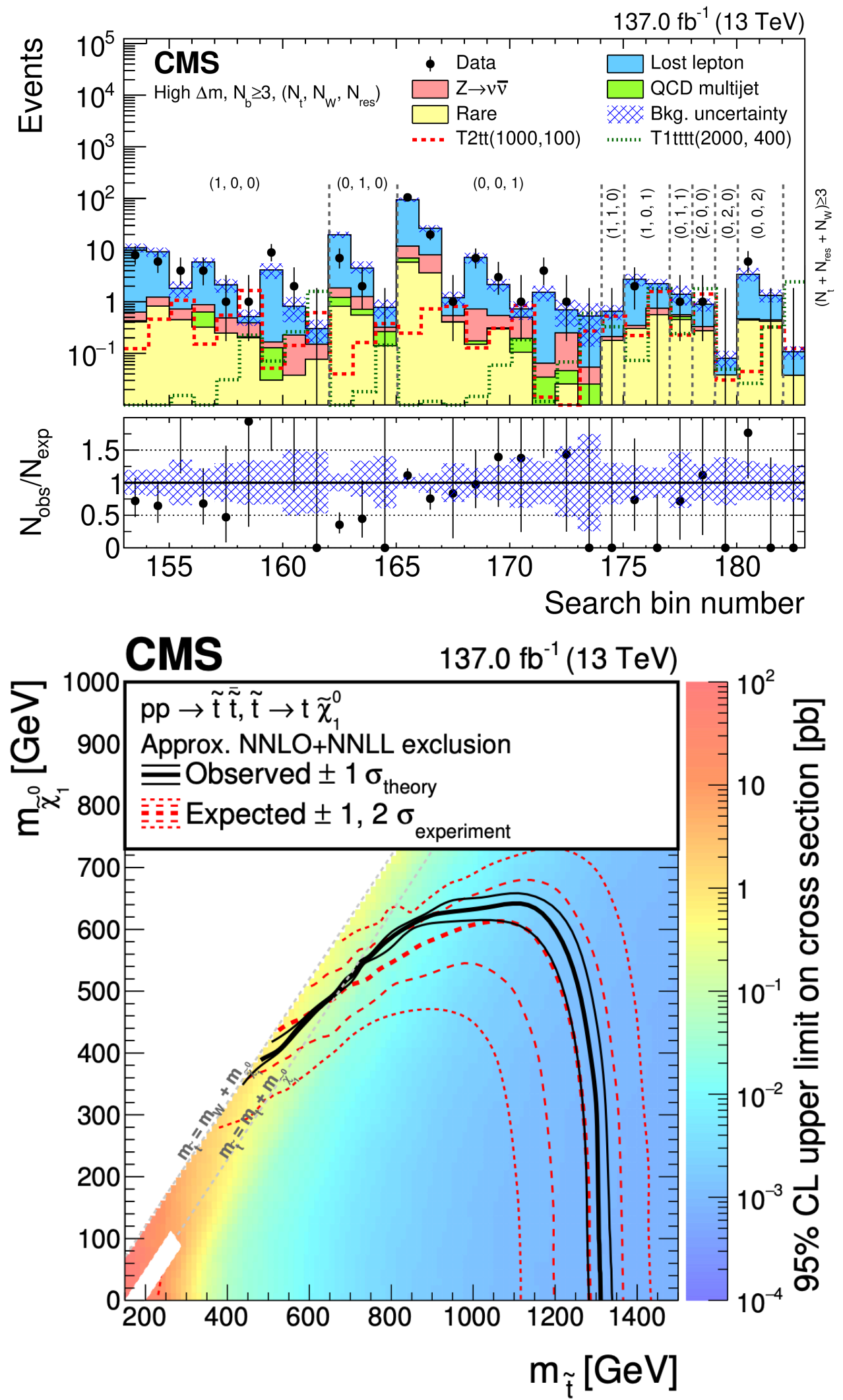

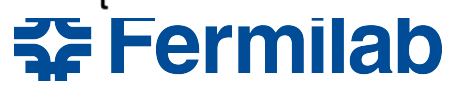




\section{Searches for stops in the final states with taus}

- Search exploiting the excellent performance of hadronic tau reconstruction

- Tracking information improving resolution at low pT by $x 2$

- Recurrent neural network algorithm to discriminate tau vs quarks and gluons, with efficiency of $75-60 \%$

- Single-tau (new) and di-tau channels

- "Stranverse mass", ETmiss, scalar sum of tau and b-jets pT

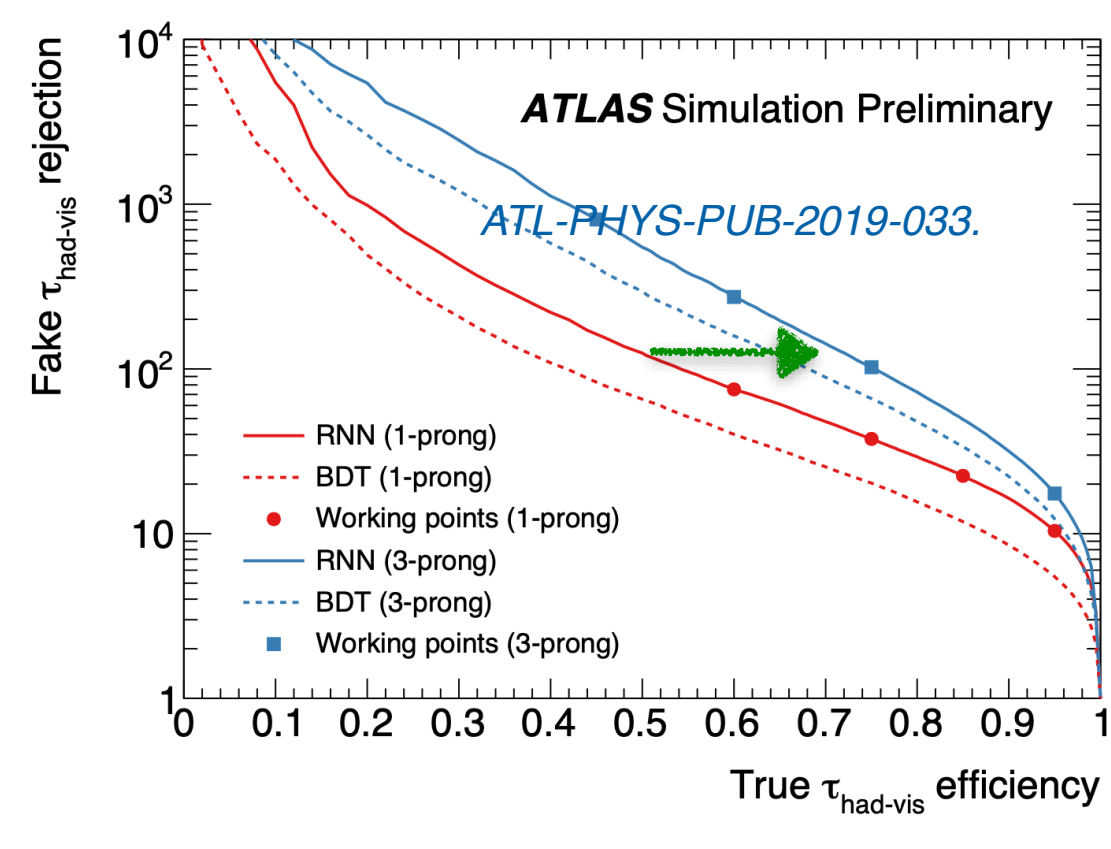
to extract signal

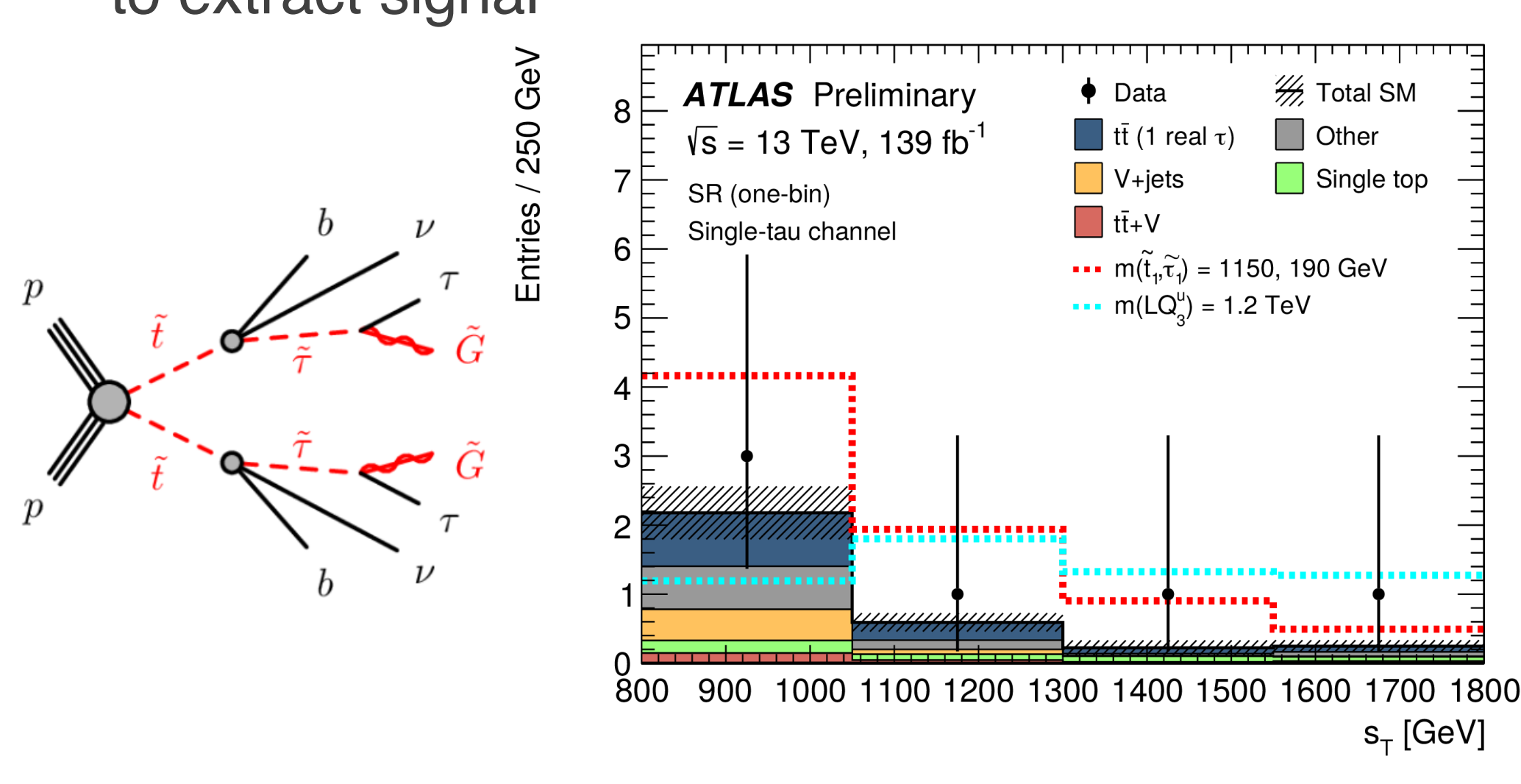

ATLAS-CONF-2021-008

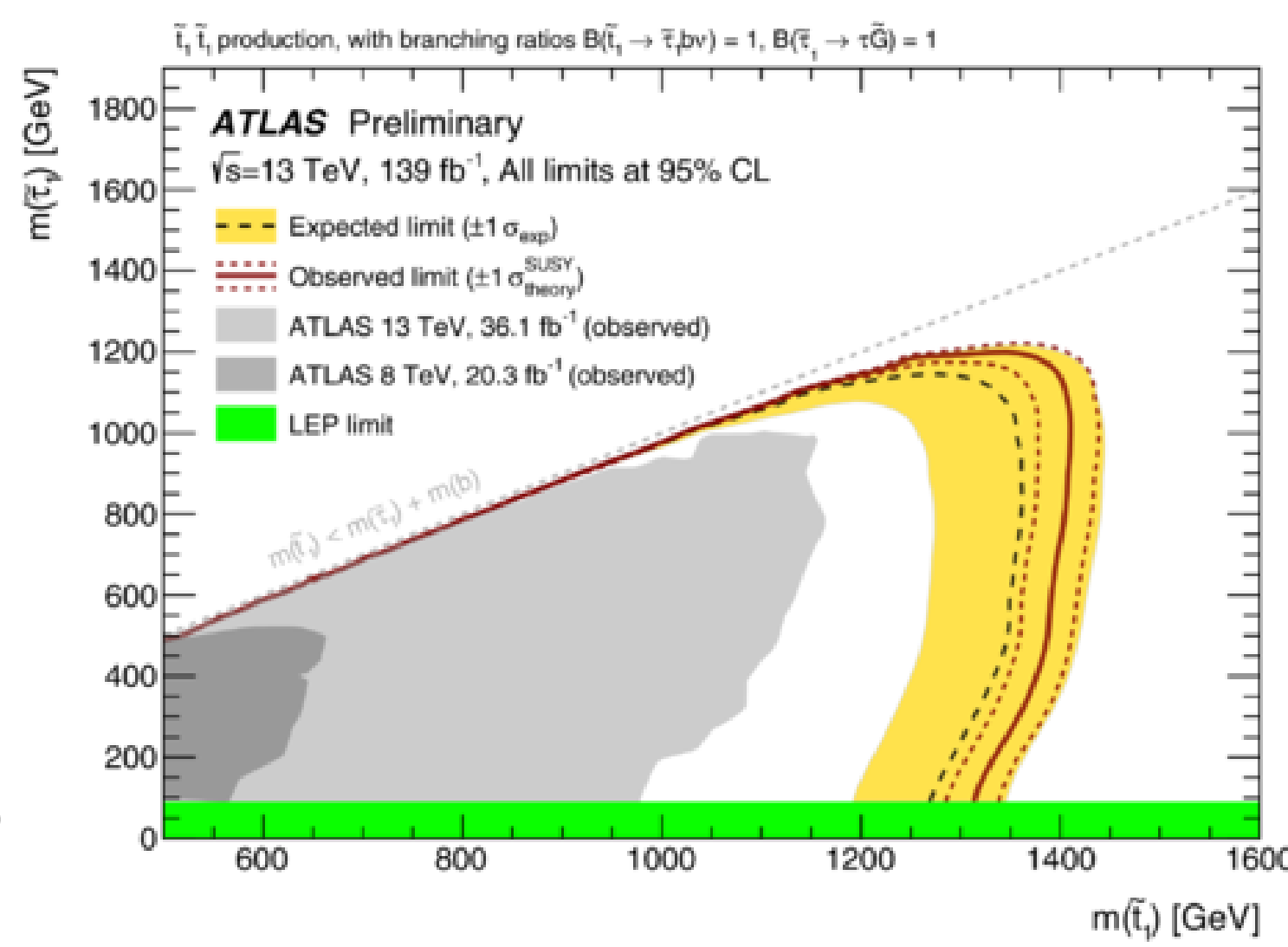

華 Fermilab 


\section{Searches for sbottoms in the final states with b-jets}

- Sensitivity of the "classic" search in the final state with two b-jets and ETmiss improved beyond luminosity scaling

- SRA for large values of $\Delta m$, larger dataset is key

- SRB targeting $\Delta m<200 \mathrm{GeV}$ using BDT-based selection

- SRC with one ISR-based selection for very compressed models (Novel b-tagger for low pT jets, 5-15 GeV)

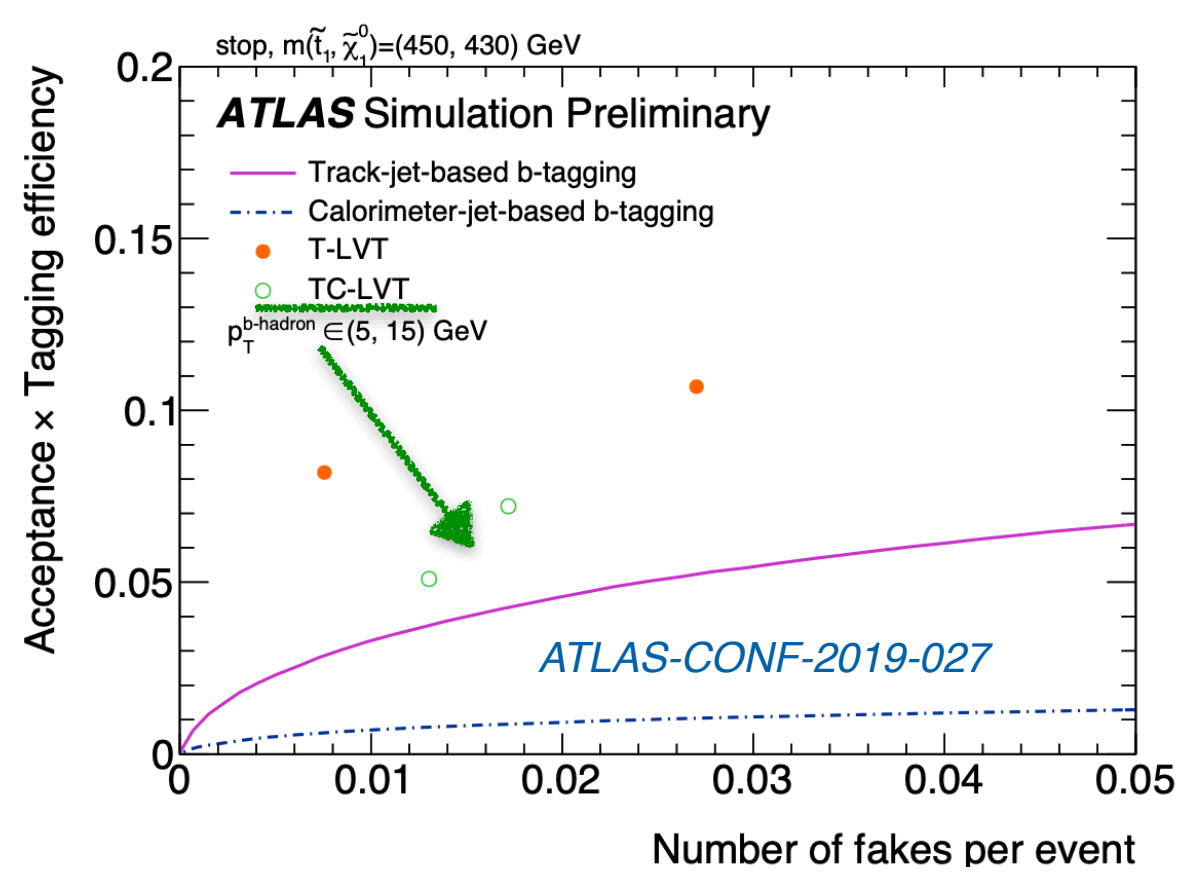

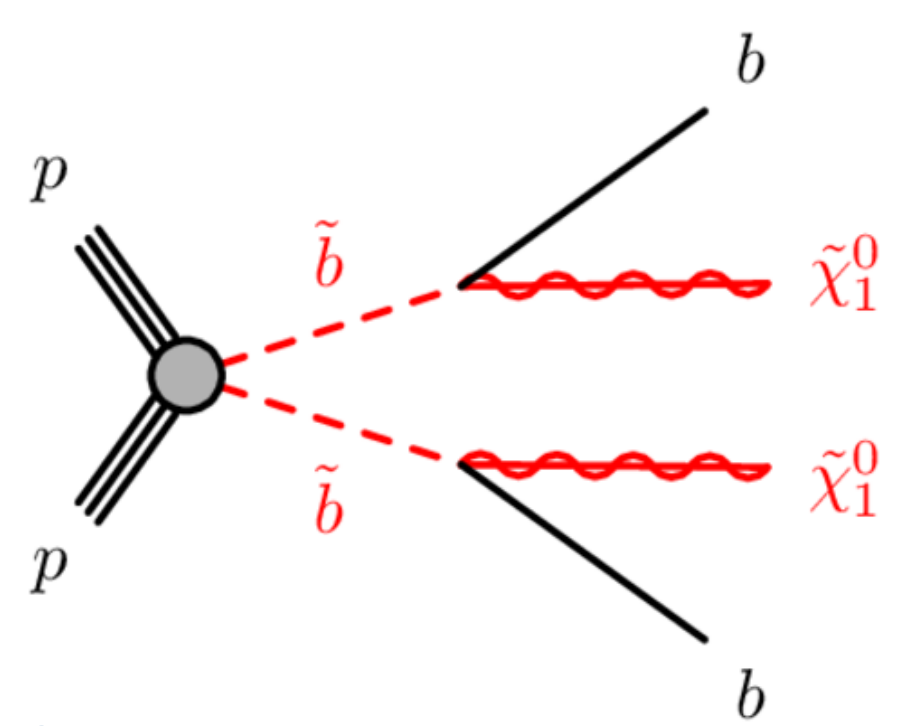

$S$ vs B discrimination via:

- mbb, transverse and cotransverse masses, ETmiss significance, scalar sums of jet $\mathrm{pT}$ and ETmiss,

- Topological observables

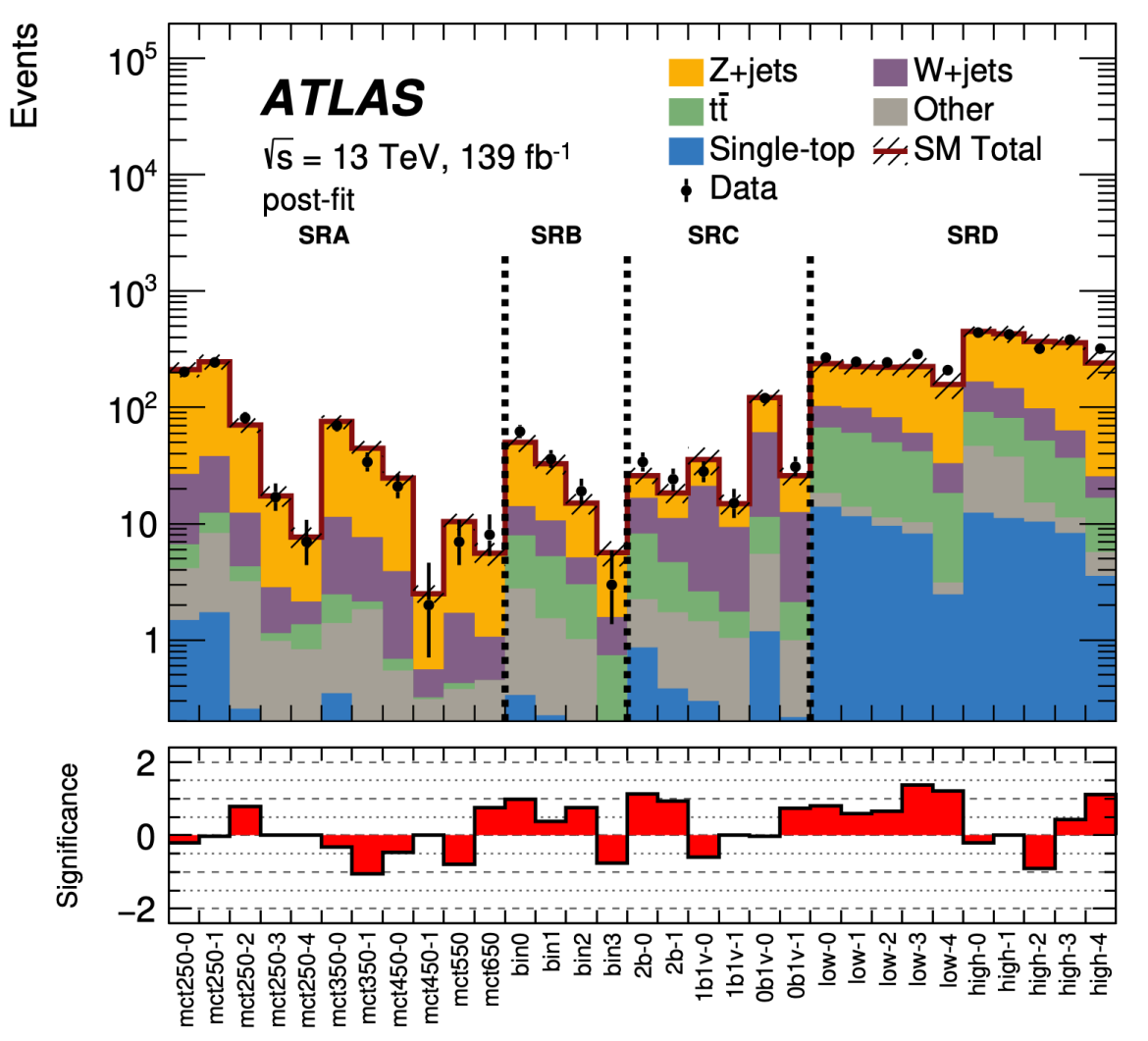

arXiv:2101.12527
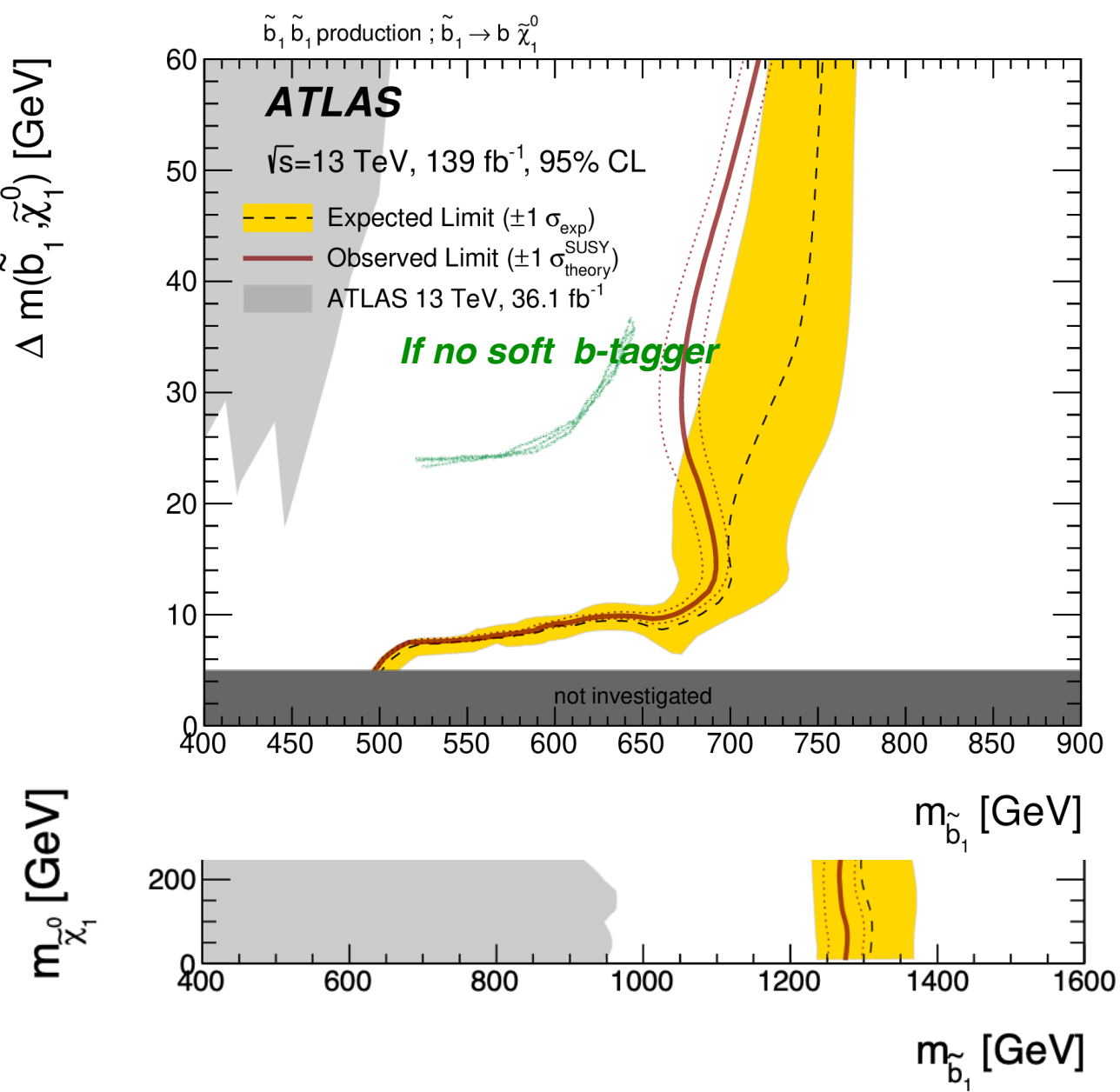

军 Fermilab 


\section{Searches for sbottoms in the final states with taus}

- Complementary search for sbottoms decaying via heavy wino-like neutralinos in final states with bs and tau lepton pairs in the Higgs mass range

- Search optimized for low mass region

- Tau leptons identified with Recurrent Neural Network

- Signal extracted from background using kinematic observables and $\Theta_{\min }$, 3D angle between taus or b-jets pairs
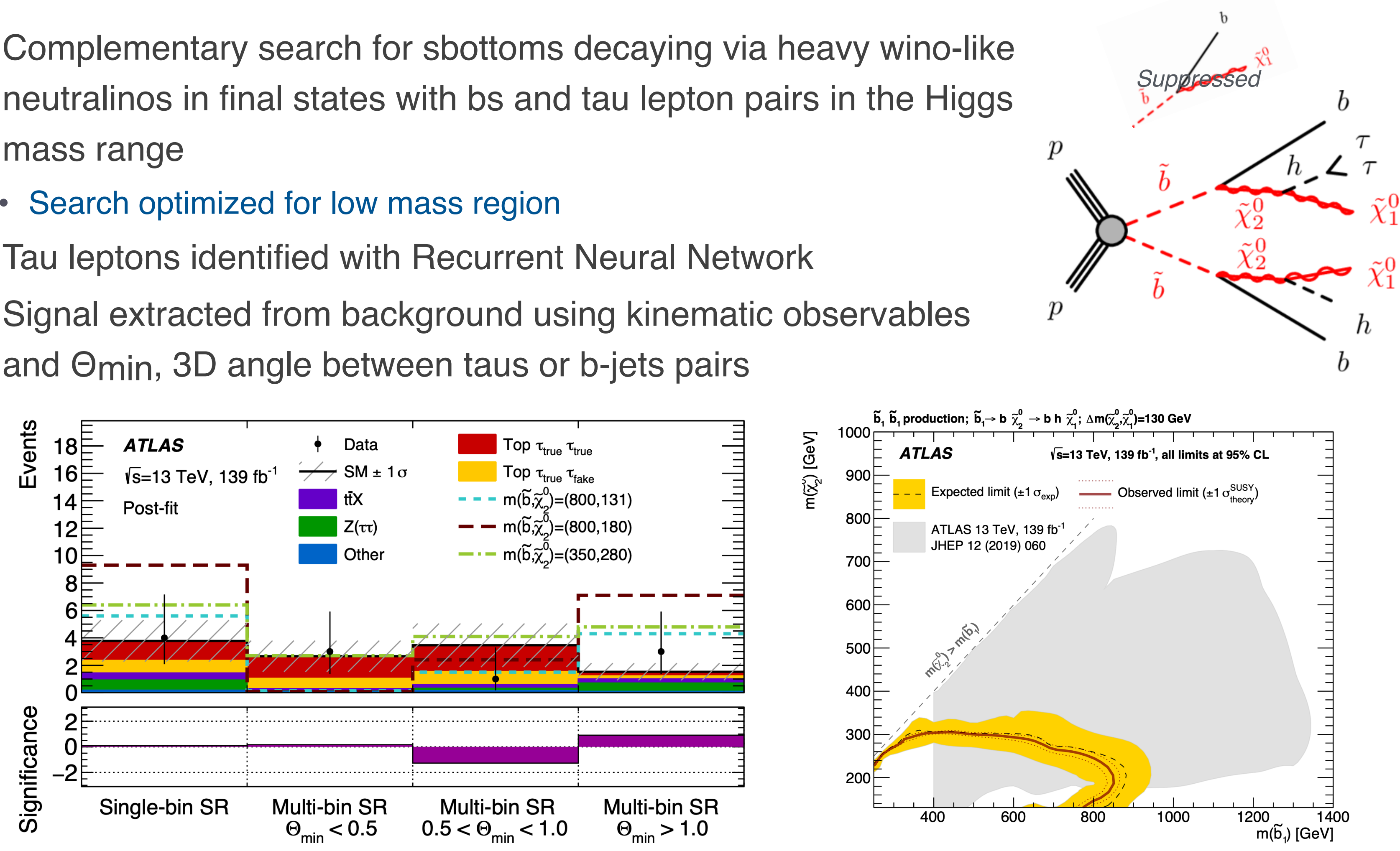


\section{Searches for Charginos and Neutralinos}




\section{Search for Charginos and Neutralinos: Multilepton Analysis}

- Classic search in "clean" leptonic final states pushed to the extreme! $p$

- Events with 2, 3, $\geq 4$ (including hadronic taus), targeting 13 decay modes

- BDT to discriminate real and fake leptons

- Parametric Neural Network using DeltaM as input for $3 \mathrm{~L}$ channel
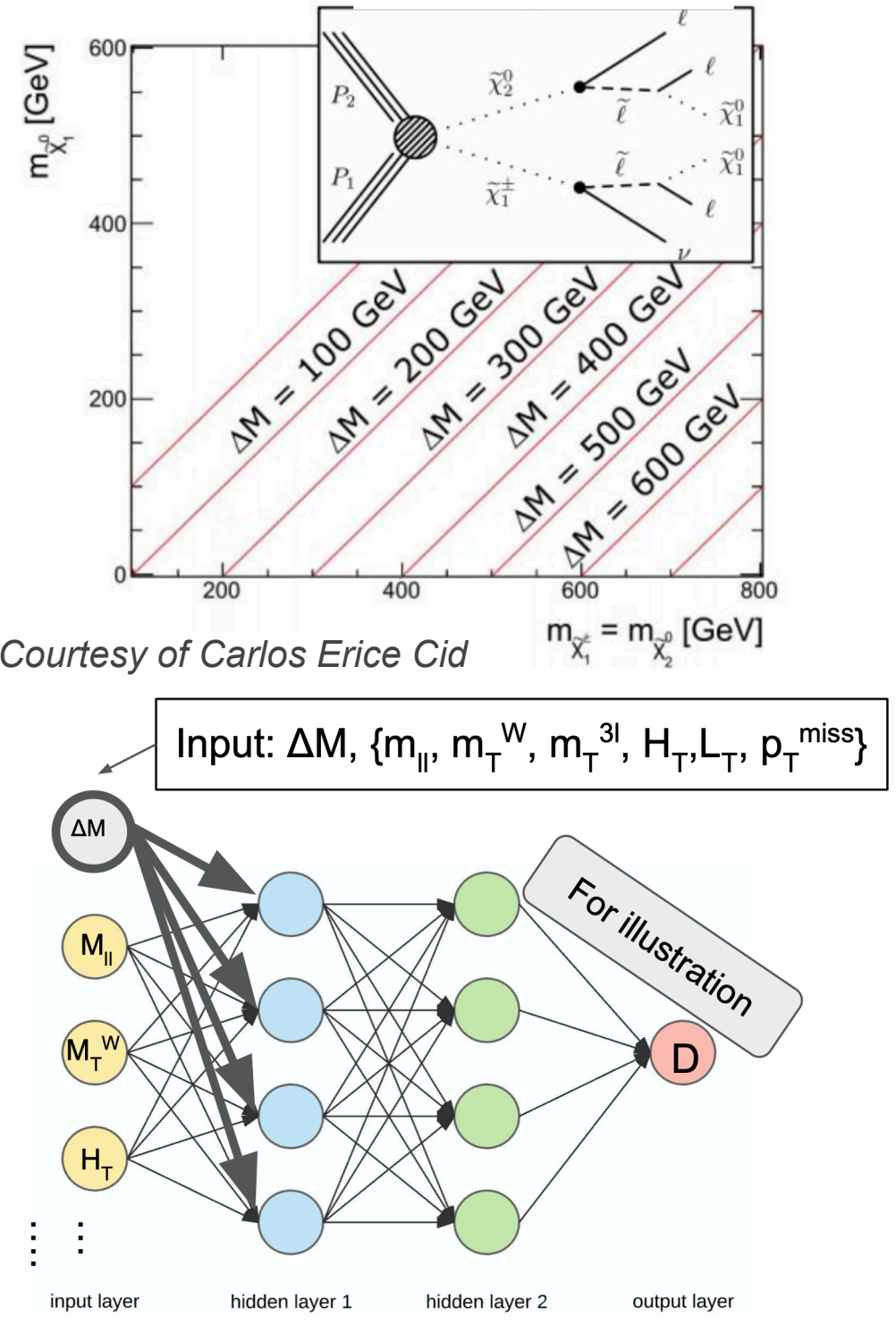

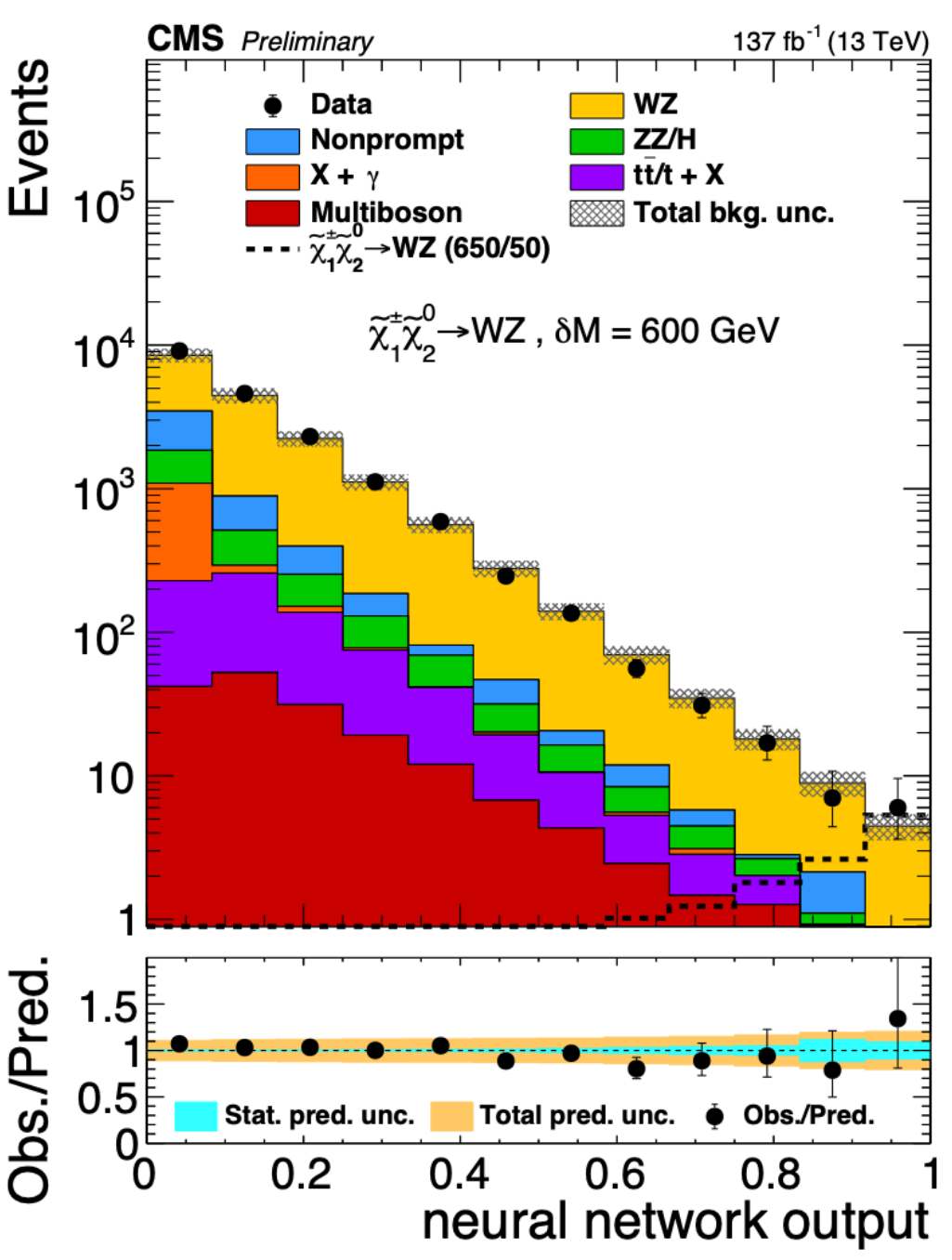

CMS-PAS-SUS-19-012

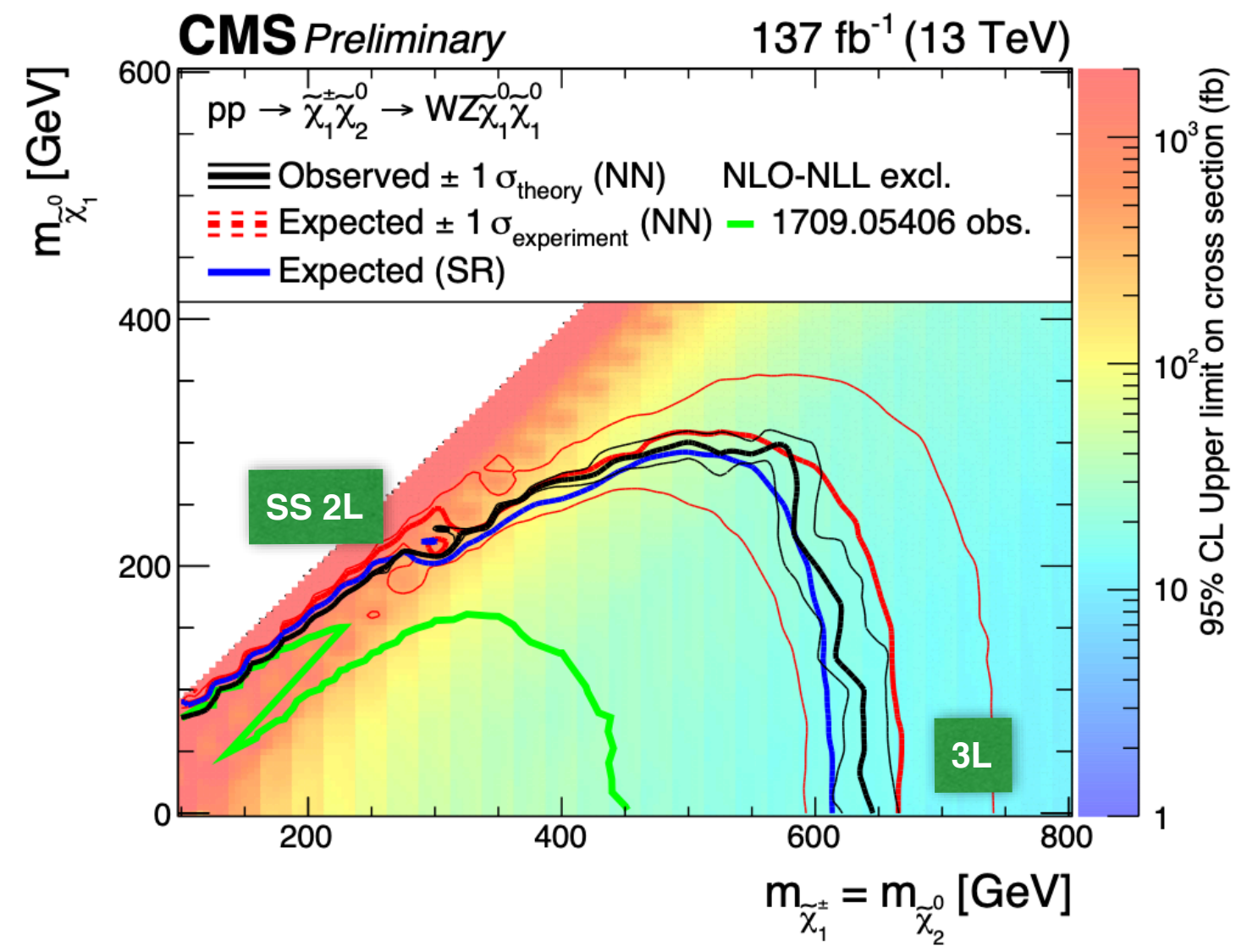

$30-40 \%$ improvement in the excluded cross sections thanks to PNN, up to $200 \%$ at low DeltaM 


\section{Search for Charginos and Neutralinos: Higgsinos}

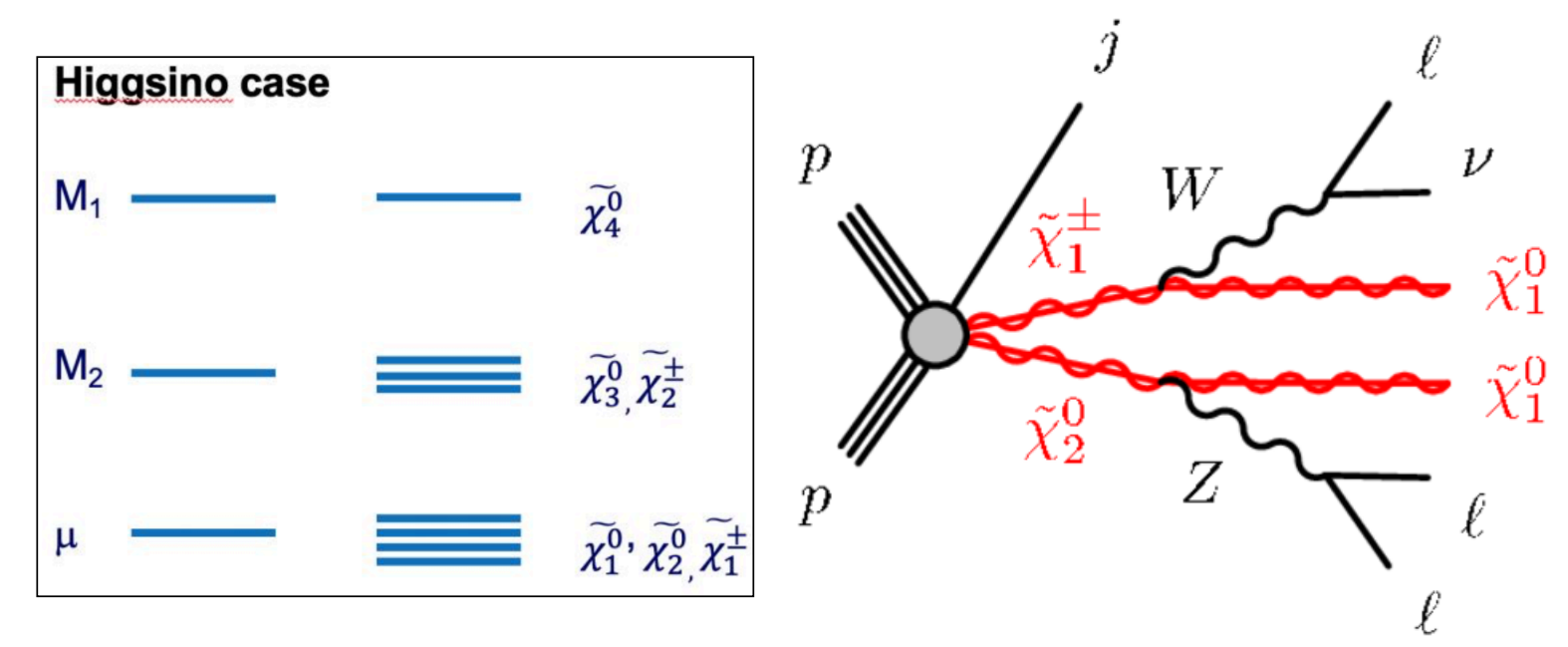

\begin{tabular}{|l|c||cc}
\hline Variable & $2 \ell$ Ewk & $3 \ell$ Ewk \\
& Low & Med/High/Ultra & Low \\
Med/High/Ultra \\
\hline \hline
\end{tabular}

$N_{\text {lep }}$
$p_{\mathrm{T}}\left(\ell_{1}\right)[\mathrm{GeV}]$ for $\mu(\mathrm{e})$

$p_{\mathrm{T}}\left(\ell_{2}\right)[\mathrm{GeV}]$ for $\mu(\mathrm{e})$

$p_{\mathrm{T}}\left(\ell_{2}\right)[\mathrm{GeV}]$ for $\mu(\mathrm{e})$
$p_{\mathrm{T}}\left(\ell_{3}\right)[\mathrm{GeV}]$ for $\mu(\mathrm{e})$

1 OS pair

1 OSSF pair

$\mathrm{M}(\ell \ell)_{S F O S}\left(\mathrm{M}(\ell \ell)_{S F O S}^{\min }\right.$ in $\left.3 \ell\right)[\mathrm{GeV}$

$\mathrm{M}(\ell \ell)_{S F O S}\left(\mathrm{M}(\ell \ell)_{S F O S}^{\text {min }}\right.$ in $\left.3 \ell\right)[\mathrm{GeV}$

$\mathrm{M}(\ell \ell)_{S F O S}\left(\mathrm{M}(\ell \ell)_{S F O S}^{\text {min }}\right.$ in $\left.3 \ell\right)[\mathrm{GeV}]$ $\mathrm{M}(\ell \ell)_{S F O S}\left(\mathrm{M}(\ell \ell)_{S F O S}^{\min }\right.$ in $\left.3 \ell\right)[\mathrm{GeV}$
$\mathrm{M}(\ell \ell)_{S F A S}^{\max }(\mathrm{AS}=$ any sign $)[\mathrm{GeV}]$ $p_{\mathrm{T}}(\ell \ell)[\mathrm{GeV}]$

$M_{T}\left(\ell_{i}, p_{\mathrm{T}}^{\text {miss }}\right)[\mathrm{GeV}](i=1,2)$

$\Delta R(\ell \ell)_{i j}(\mathrm{i}, \mathrm{j}=1,2,3)$

$p_{\mathrm{T}}^{\text {miss }}[\mathrm{GeV}]$

$p_{\mathrm{T}}^{\text {miss,corr }}[\mathrm{GeV}]$

$H_{\mathrm{T}}[\mathrm{GeV}]$

$p_{\mathrm{T}}^{\text {miss }} / H_{\mathrm{T}}$

Jet ID tight WP for leading jet

$\mathrm{N}_{b}\left(p_{\mathrm{T}}>25 \mathrm{GeV}\right)$

$M_{\tau \tau}$

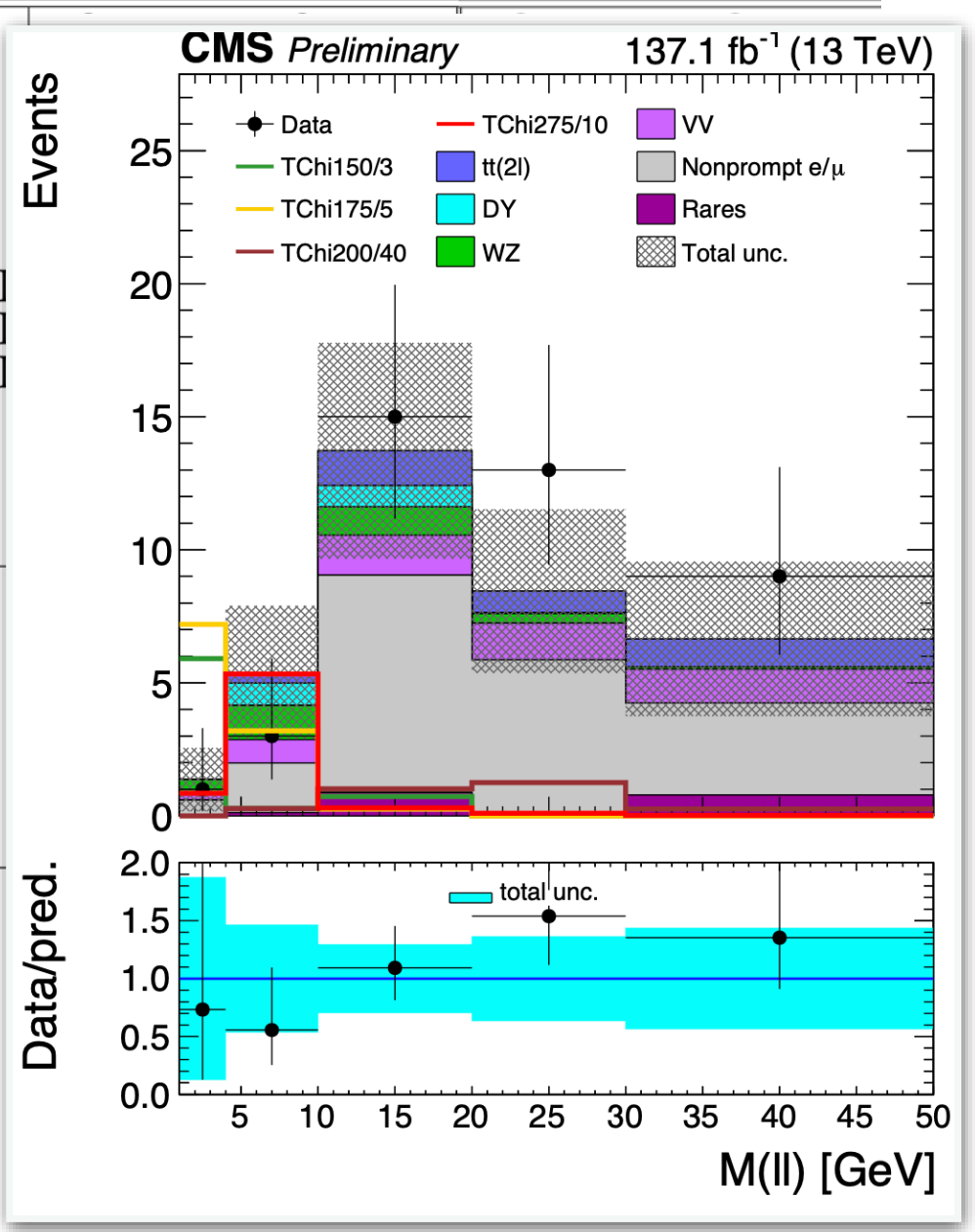

- Higgsino-like EWK-inos in the final state with one jet, moderate ETmiss and 2/3 soft leptons

- Muon (electron) pT > 3.5 (5) GeV

- Dilepton invariant mass down to $1 \mathrm{GeV}$

- Events classified based on ETmiss

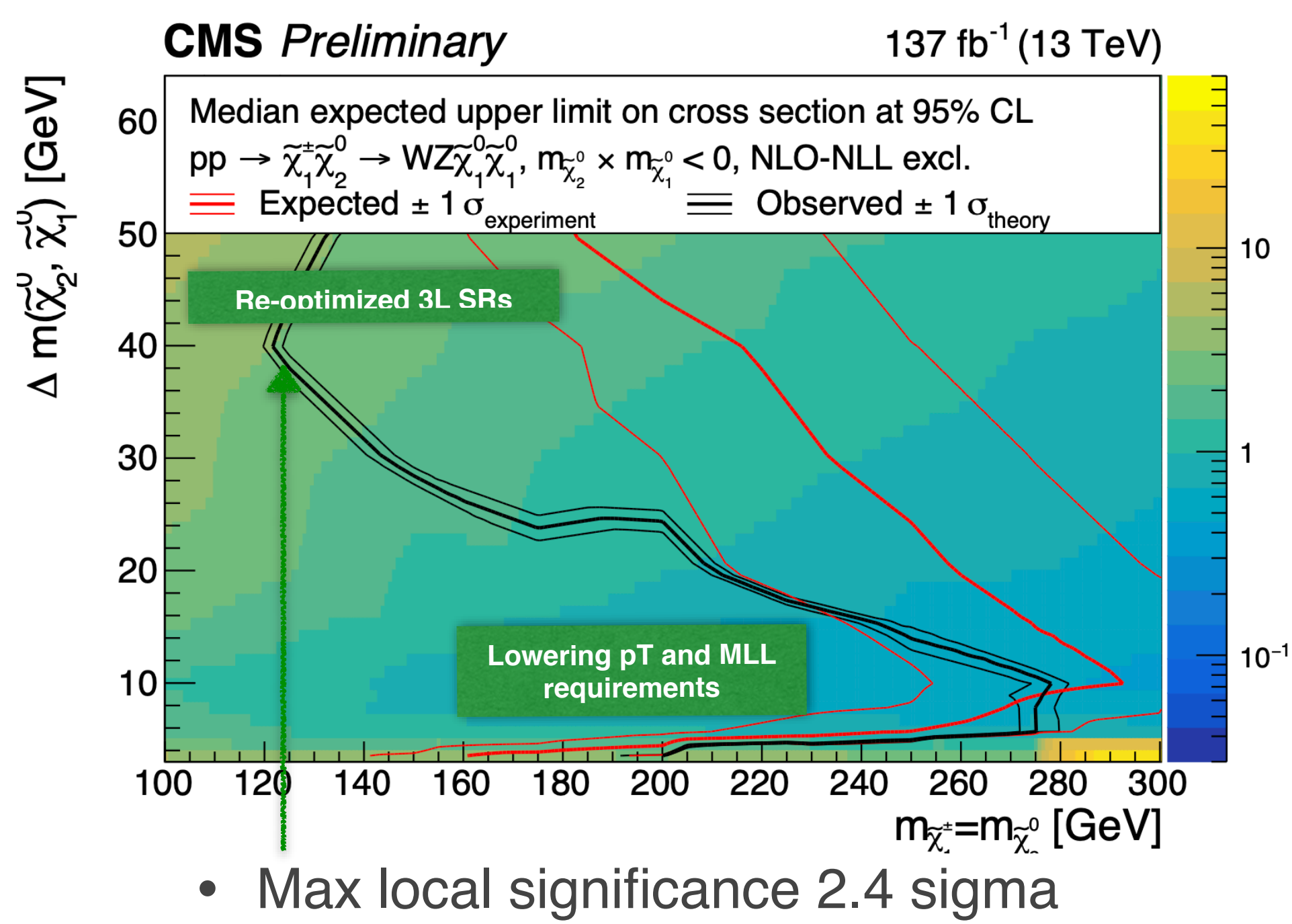




\section{Search for long-lived charginos}

- Charginos decaying as $\tilde{\chi}^{ \pm} \rightarrow \pi^{ \pm} \chi^{0}$ expected in models with very compressed spectra

- Striking signature with a 'disappearing track'

- Pixel tracklets with no hits in the outermost silicon trackers and no calorimeter activity (newly introduced requirement)

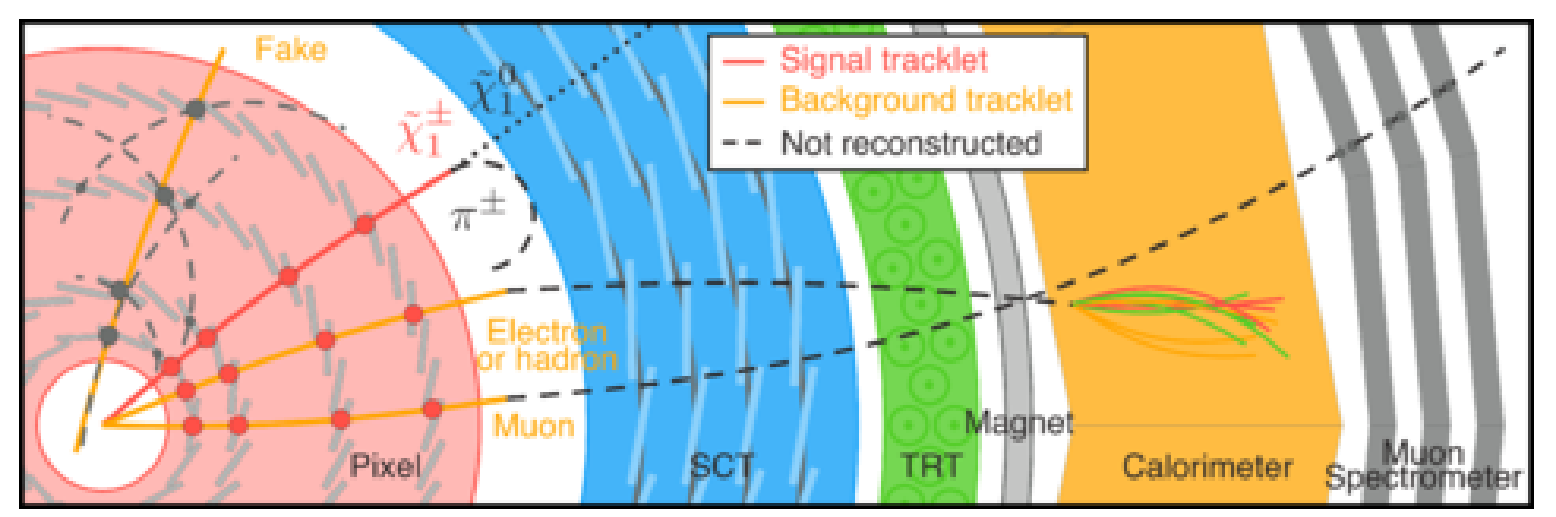

- ETmiss and number of jets for $\mathrm{S}$ to $\mathrm{B}$ discrimination
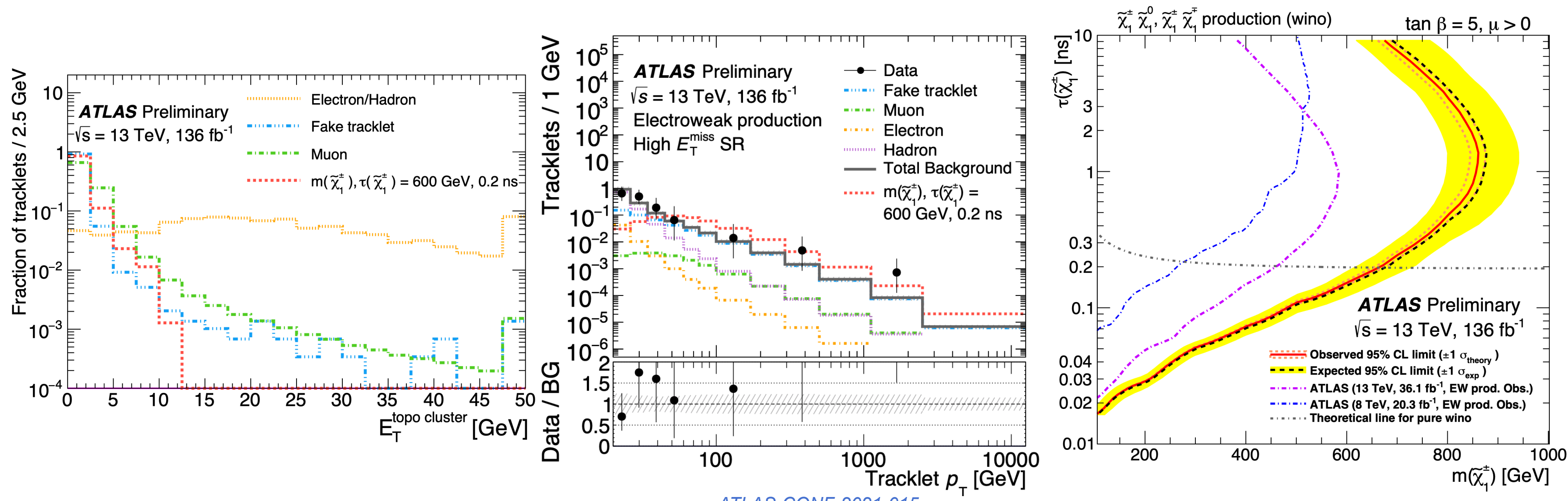

ATLAS-CONF-2021-015 


\section{Other Searches for Long Lived SUSY}

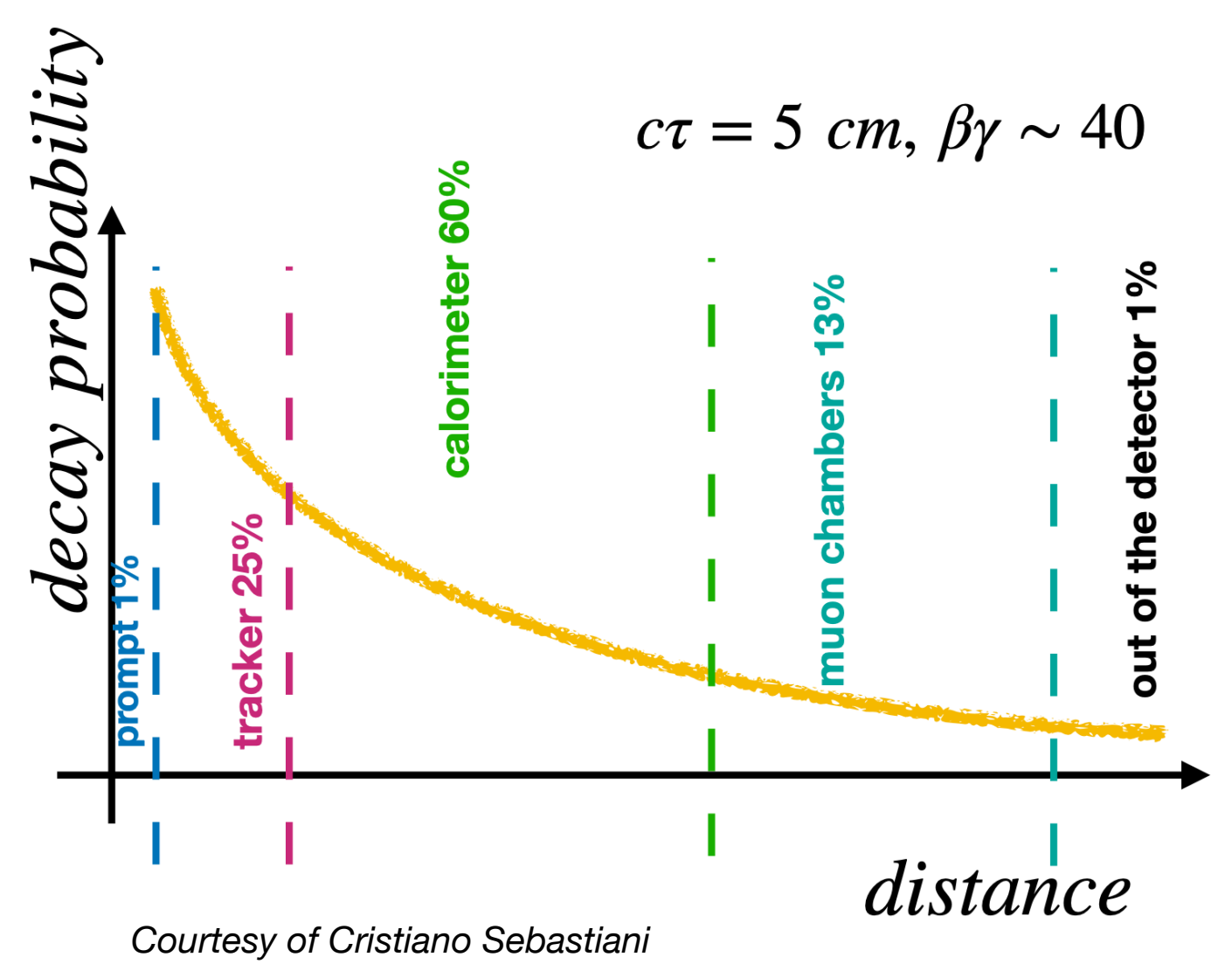




\section{Long-lived particles decaying to jets with displaced vertices}

- (Model independent) search for long-lived particles decaying into multiple jets

- Unique signature with 2 multi-track vertices displaced from the interaction point but within the beam pipe

- Custom vertex reconstruction

- Fit to vertices separation in events with large hadronic scalar energy
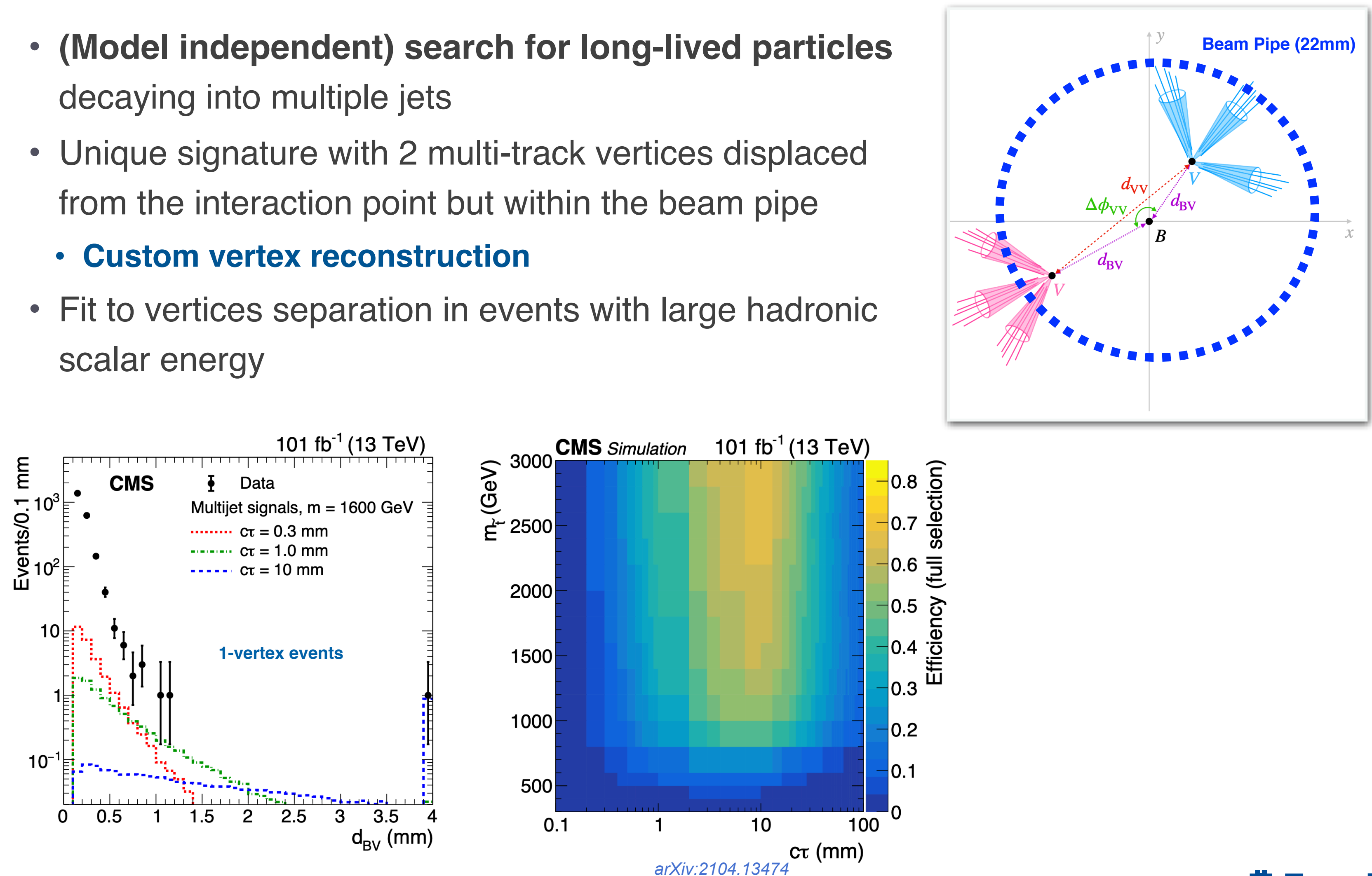


\section{Long-lived particles decaying to jets with displaced vertices}

- (Model independent) search for long-lived particles decaying into multiple jets

- Unique signature with 2 multi-track vertices displaced from the interaction point but within the beam pipe

- Custom vertex reconstruction

- Fit to vertices separation in events with large hadronic

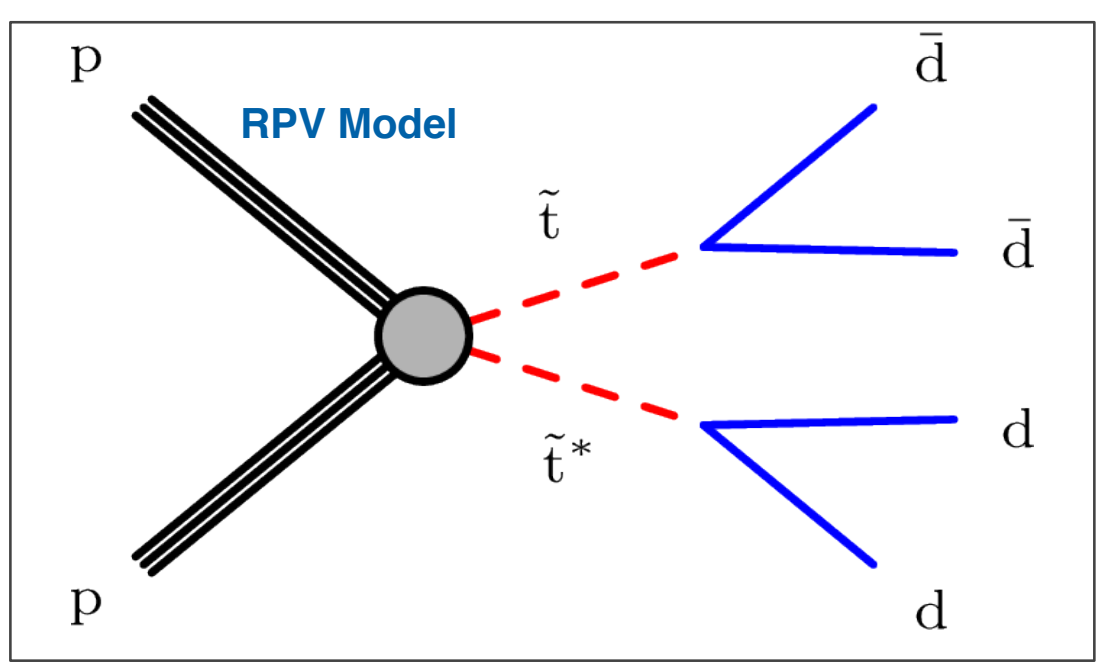
scalar energy
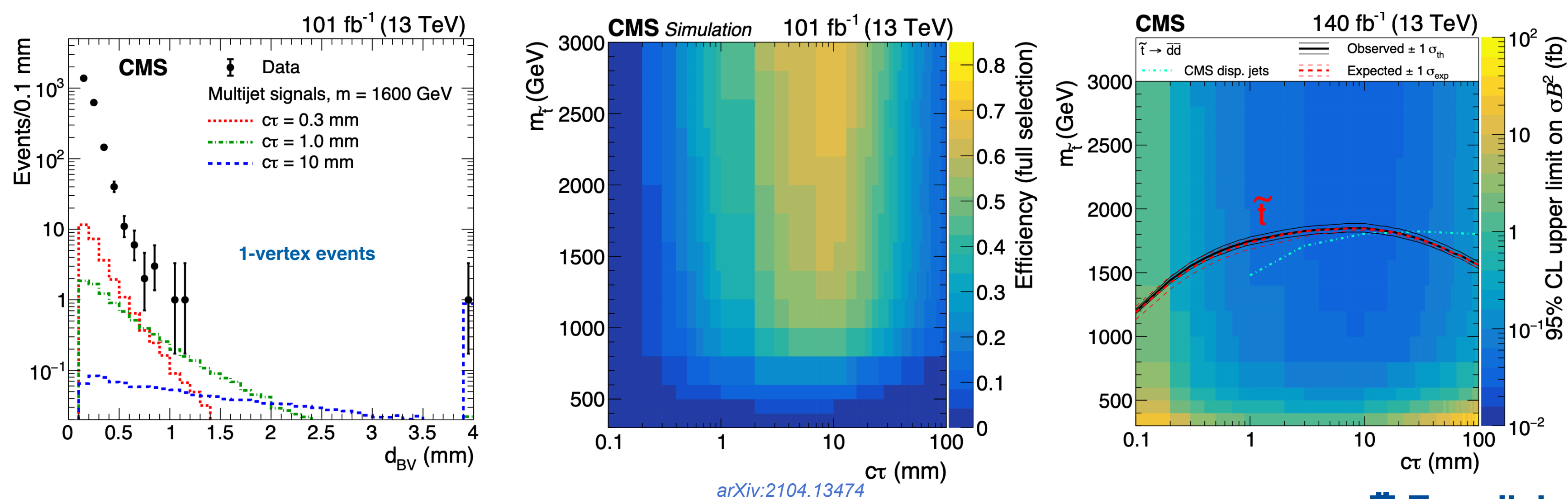


\section{Search for the decays of stopped long-lived particles}

- Long-lived particles can sit in the detector before decaying in jets leading to a unique signatures with out of time energy deposits in calorimeter

- Events with ETmiss and jets collected during the "empty" bunch crossings (total dataset of 600 hours!)

- Challenging estimate of unusual backgrounds from cosmic rays, beam induced background, cavern background, calorimeter noise
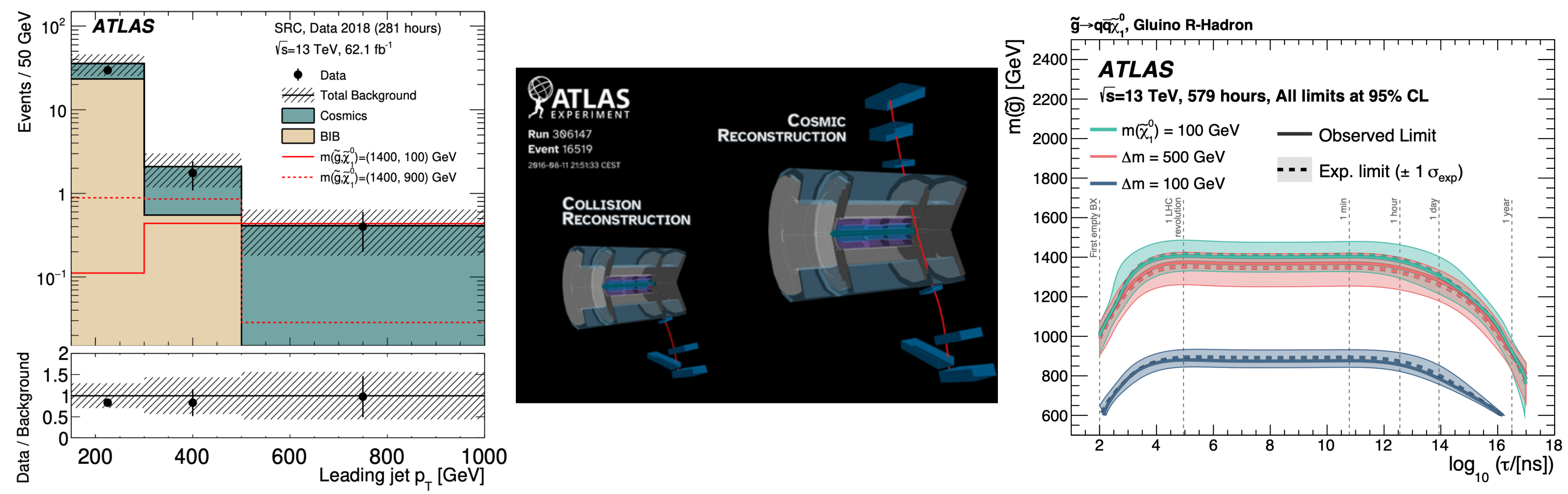


\section{Searches for RPV and Stealth SUSY}




\section{RPV SUSY in events with multiple leptons}

$$
W_{\mathrm{RPV}}=\frac{1}{2} \lambda_{i j k} L_{i} L_{j} E_{k}^{c}+\lambda_{i j k}^{\prime} L_{i} Q_{j} D_{k}^{c}+\frac{1}{2} \lambda_{i j k}^{\prime \prime} U_{i}^{c} D_{j}^{c} D_{k}^{c}
$$

- Inclusive searches for gluinos, sleptons, charginos in final states with small ETmiss
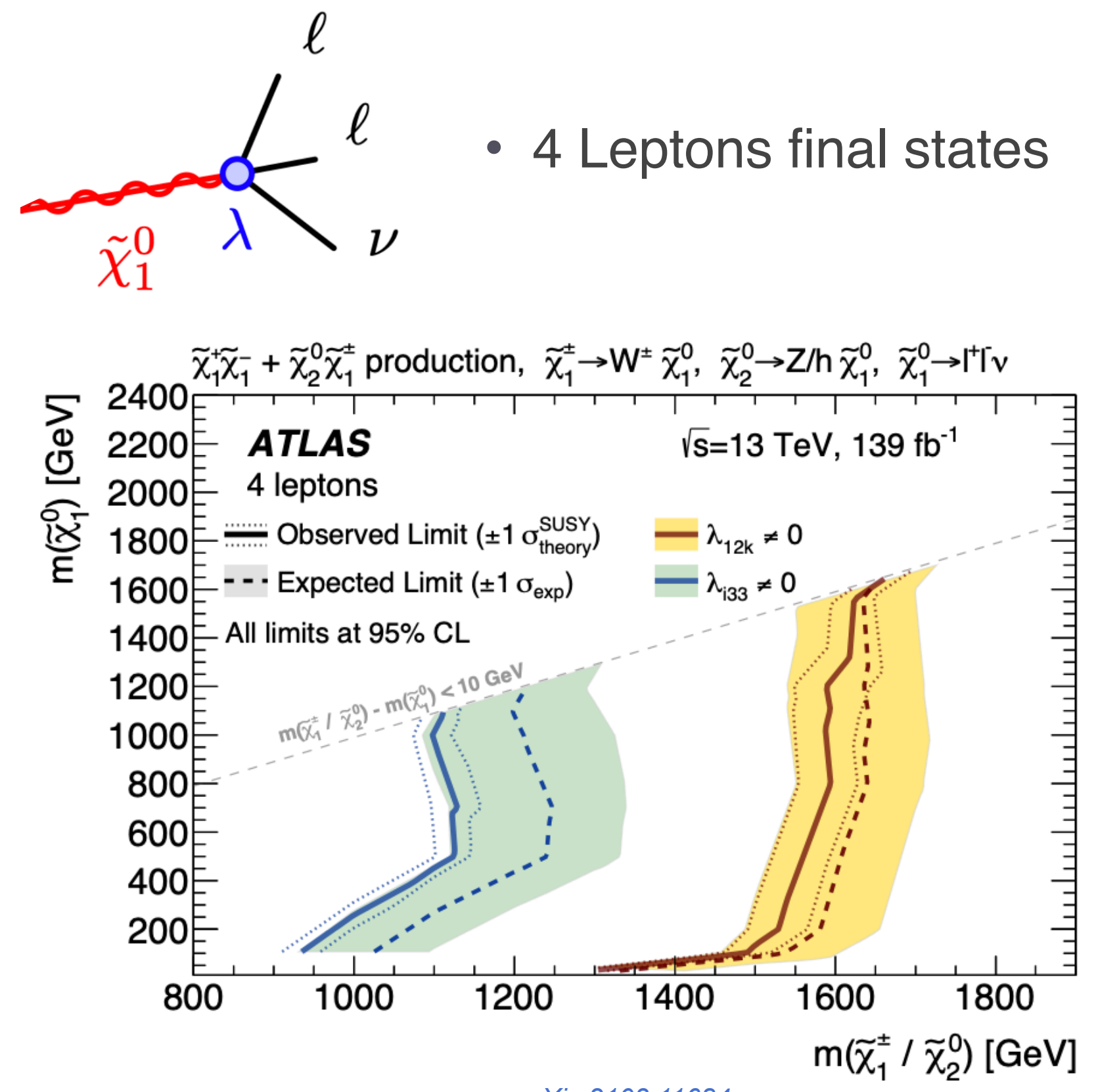

- 4 Leptons final states

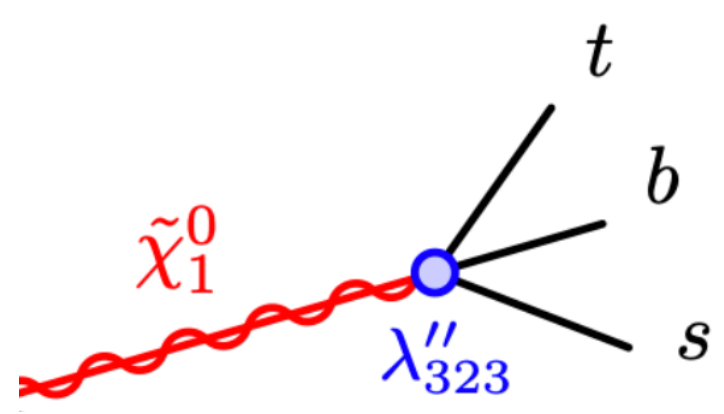

1 lepton and (b-)jets

- Adversarial training of NN to select higgsinos

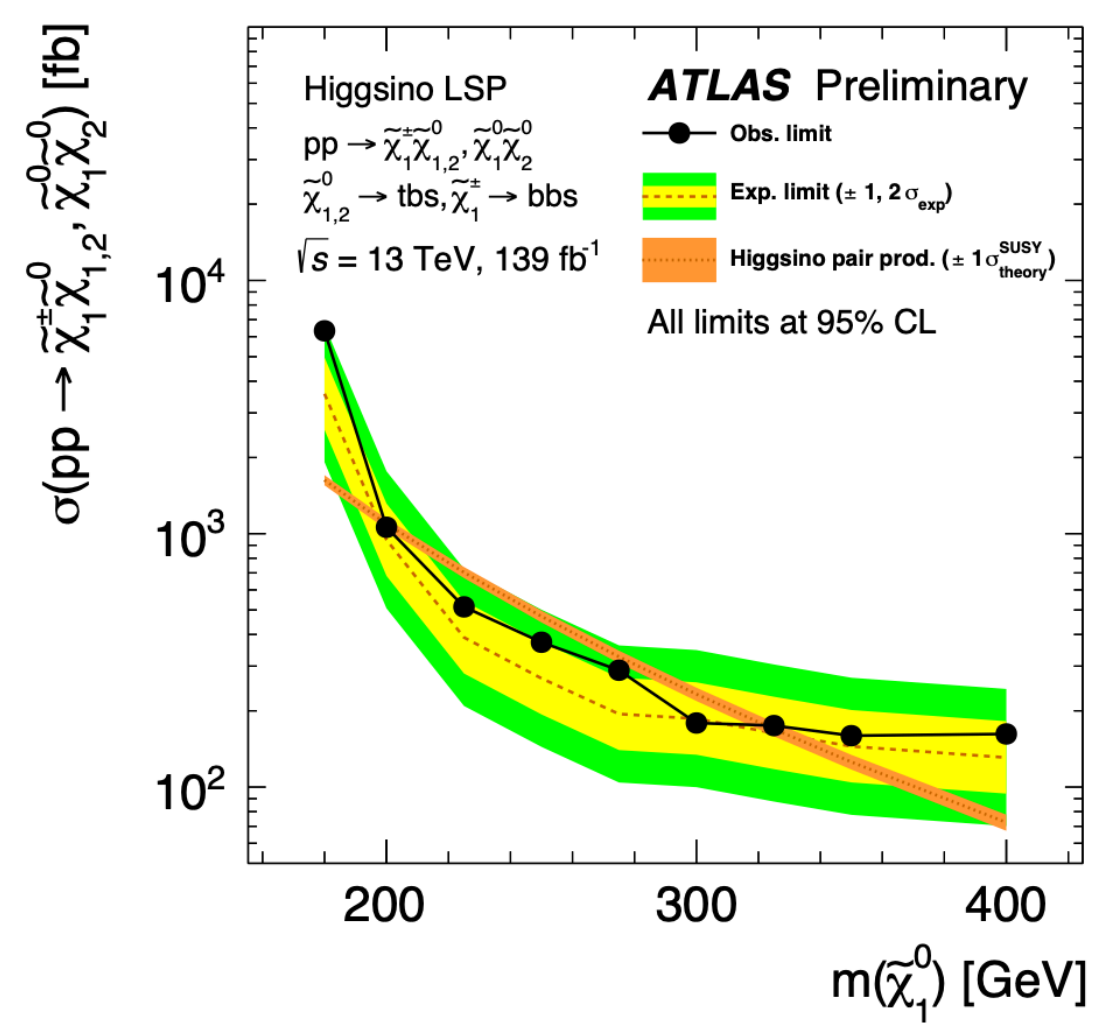

ATLAS-CONF-2021-007 


\section{Searches for Stealth SUSY in the stop sector}

First LHC search

of this kind

- New hidden "stealth" sector of light particles with small or absent couplings to the SUSY breaking sector and finite couplings to the visible sector

- SUSY approximately conserved, stealth particles remaining nearly mass-degenerate with their superpartners

- Challenging final states with one lepton, no ETmiss, $\geq 8$ jets

- Neural Network with gradient reversal to discriminate S vs. B

- 2D fit to NN score and Njet to extract signal

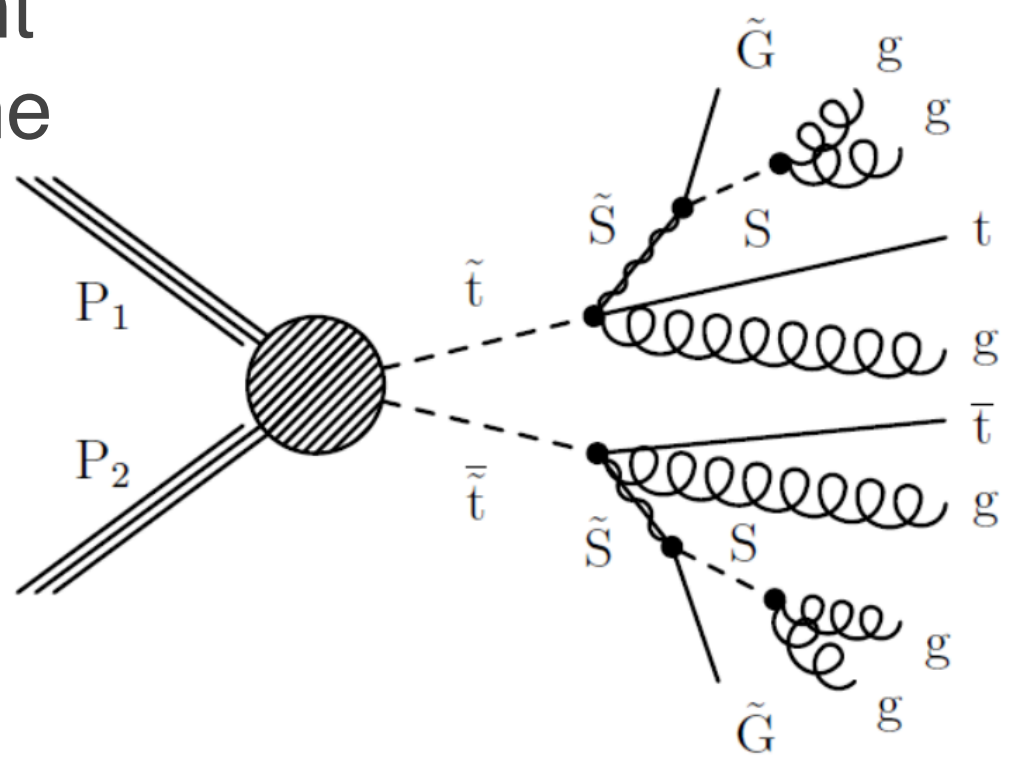

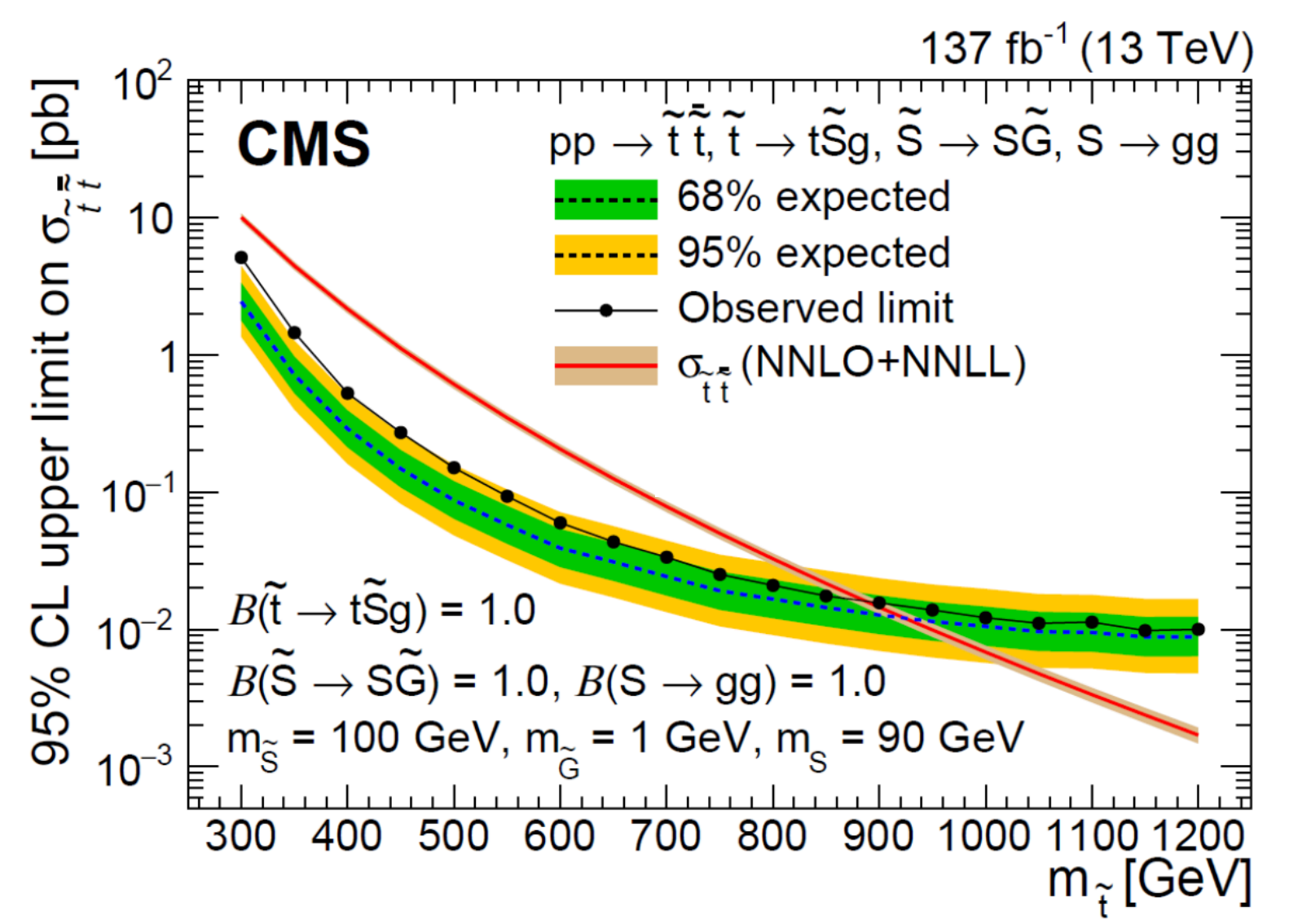

arxXiv:2102.06976
- $2.8 \sigma$ local significance for 400GeV

- Sensitivity to RPV decays of stops up to $\sim 700 \mathrm{GeV}$ 


\section{Outlook}

- At the LHC, SUSY can manifest itself in a variety of (very challenging) signatures

- High and low ETmiss, high and low momentum objects, prompt and displaced objects, low and high object multiplicity, in time and out of time signals, ....

- The ATLAS and CMS experiments have developed a broad and sophisticated search in Run 2 data adopting breakthrough experimental techniques

- Pushing the sensitivity for gluinos, stops, and EWK-inos as high as $\sim 2,1,0.8 \mathrm{TeV}$ scales for models with R-parity conservation

- Shifting paradigm to probe for unconventional signatures expected in Long Lived, R-parity violation, Stealth, ... SUSY

- Special datasets collected with novel triggers remain to be explored

- Run 3 is around the corner, with great potential for discovery thanks to new capabilities of the upgraded ATLAS and CMS detectors

The search continues! 


\section{Additional material}




\section{ATLAS and CMS at Run 2}

- Run-2 represents an unprecedented dataset for HEP

- Outstanding understanding of detector performance and simulation at fundamental levels

- Breakthrough triggers pushing the reach of the experiments

- Reading out L1 triggered events, x10 more events (scouting, trigger level analysis)

- "Parking" unbiased set of B-decays events (10B events in Run2)

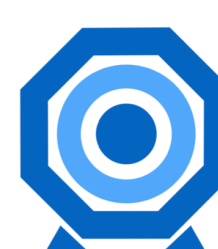

$40 \mathrm{mHz}$

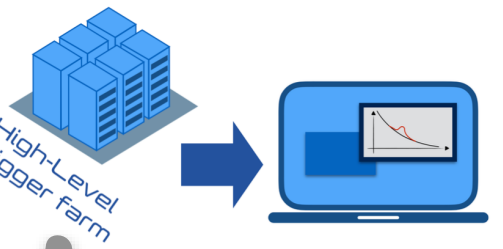

New events

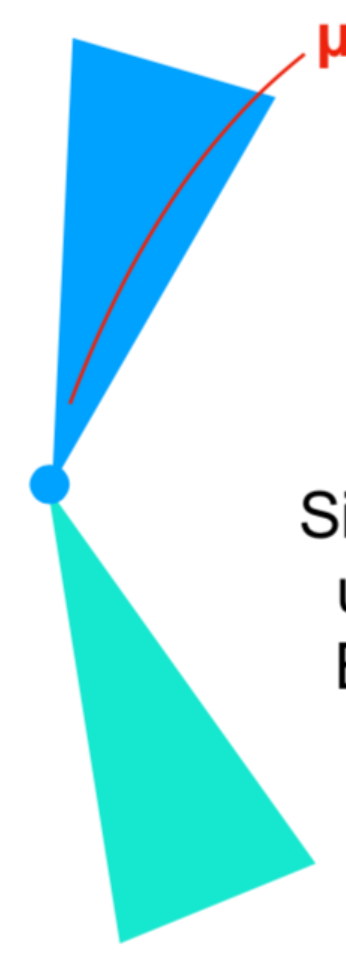

Tag-side:

$b \rightarrow \mu X$

Signal-side: unbiased B decays
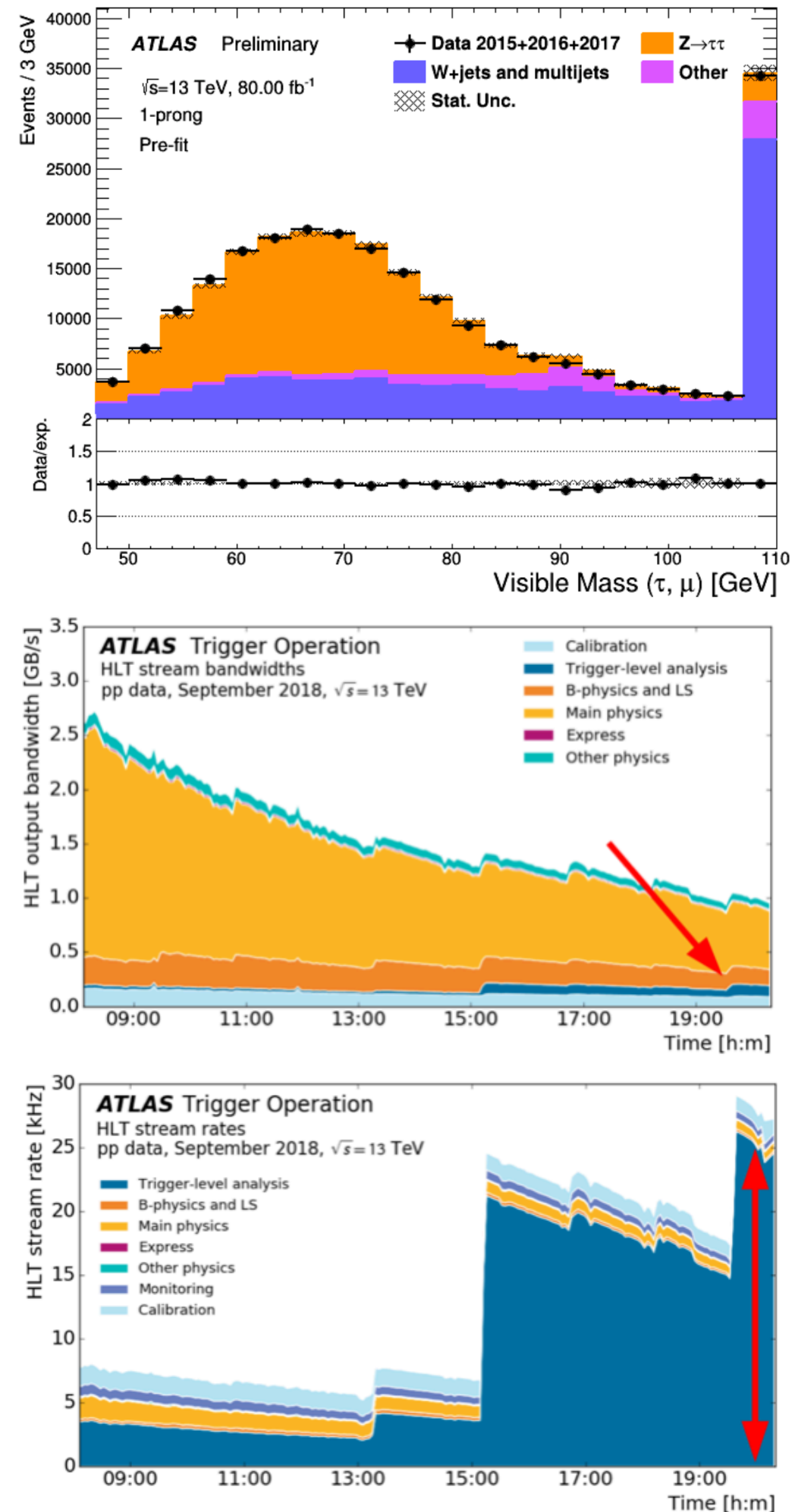


\section{Searches for stops directly produced and in gluino decays}

- Parametric DNN based selection of events with two leptons, b-jets and ETmiss to probe the "corridor"

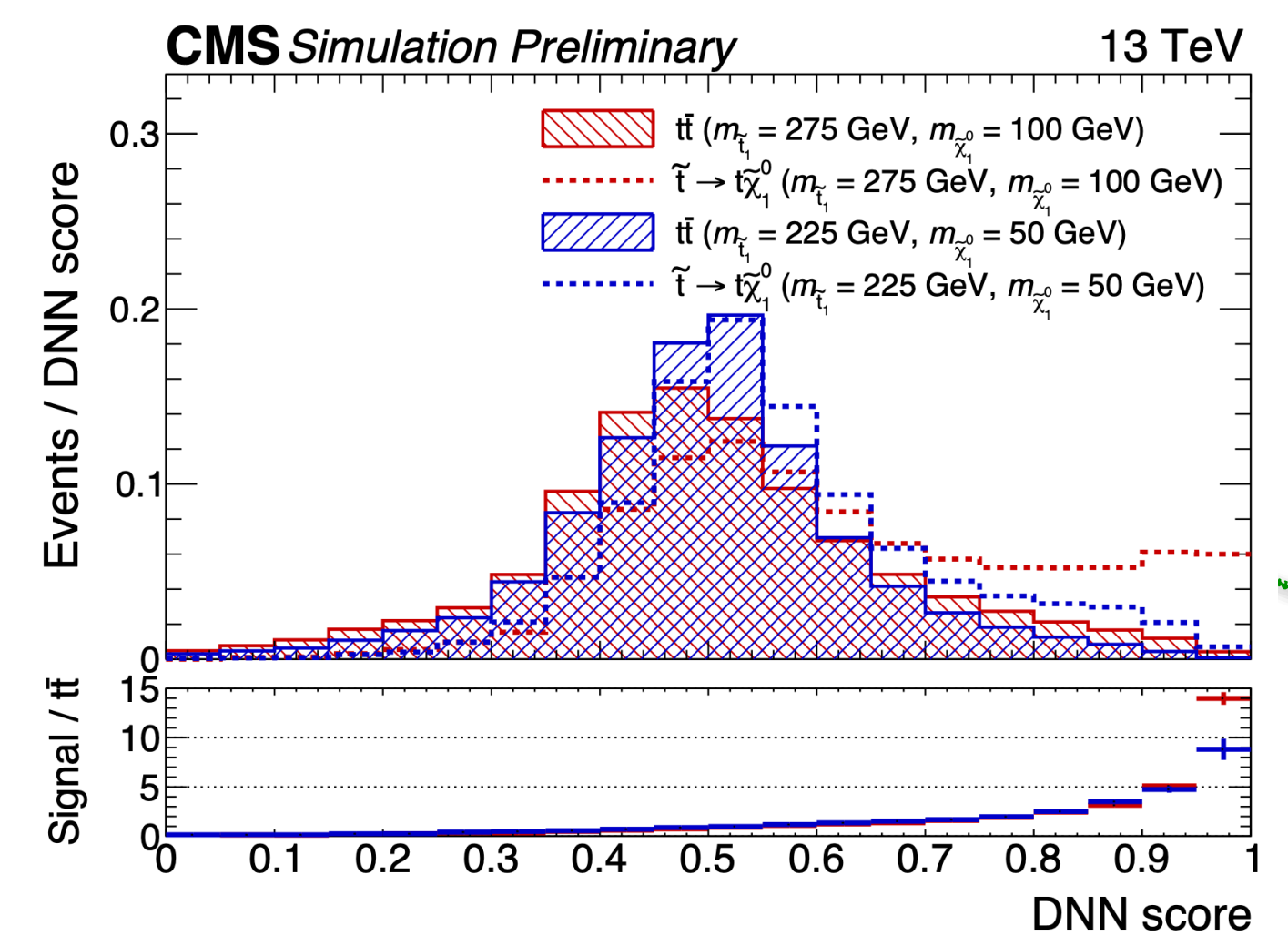

CMS Preliminary $137 \mathrm{fb}^{-1}(13 \mathrm{TeV})$

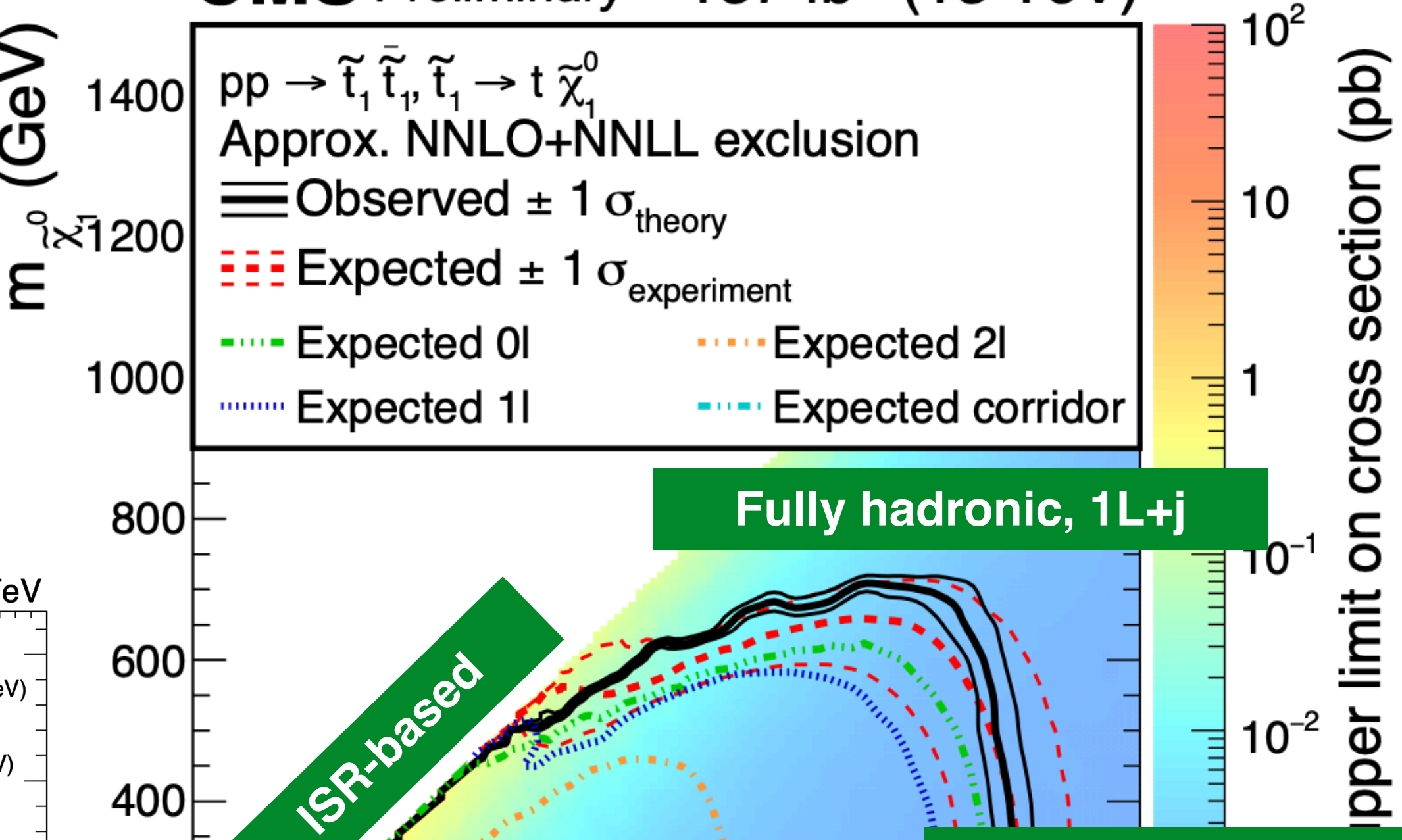

Fully hadronic Boosted tops and bosons

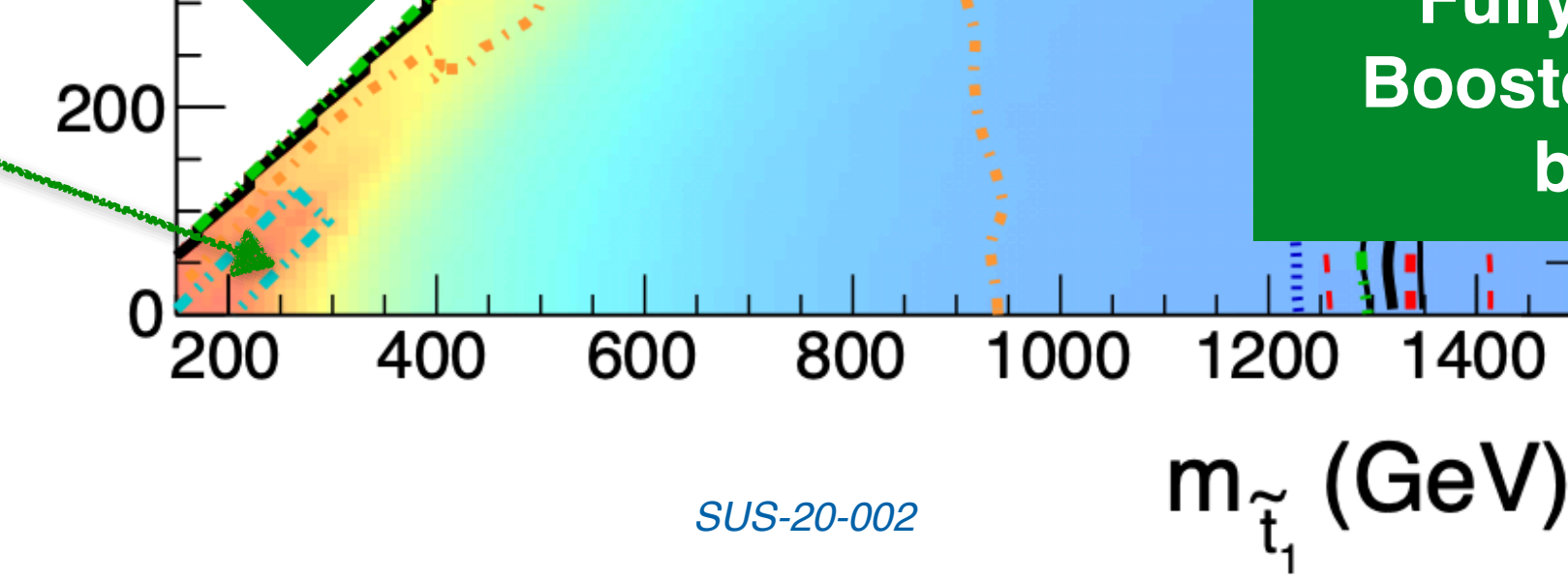




\section{Search for Charginos and Neutralinos: pMSSM}

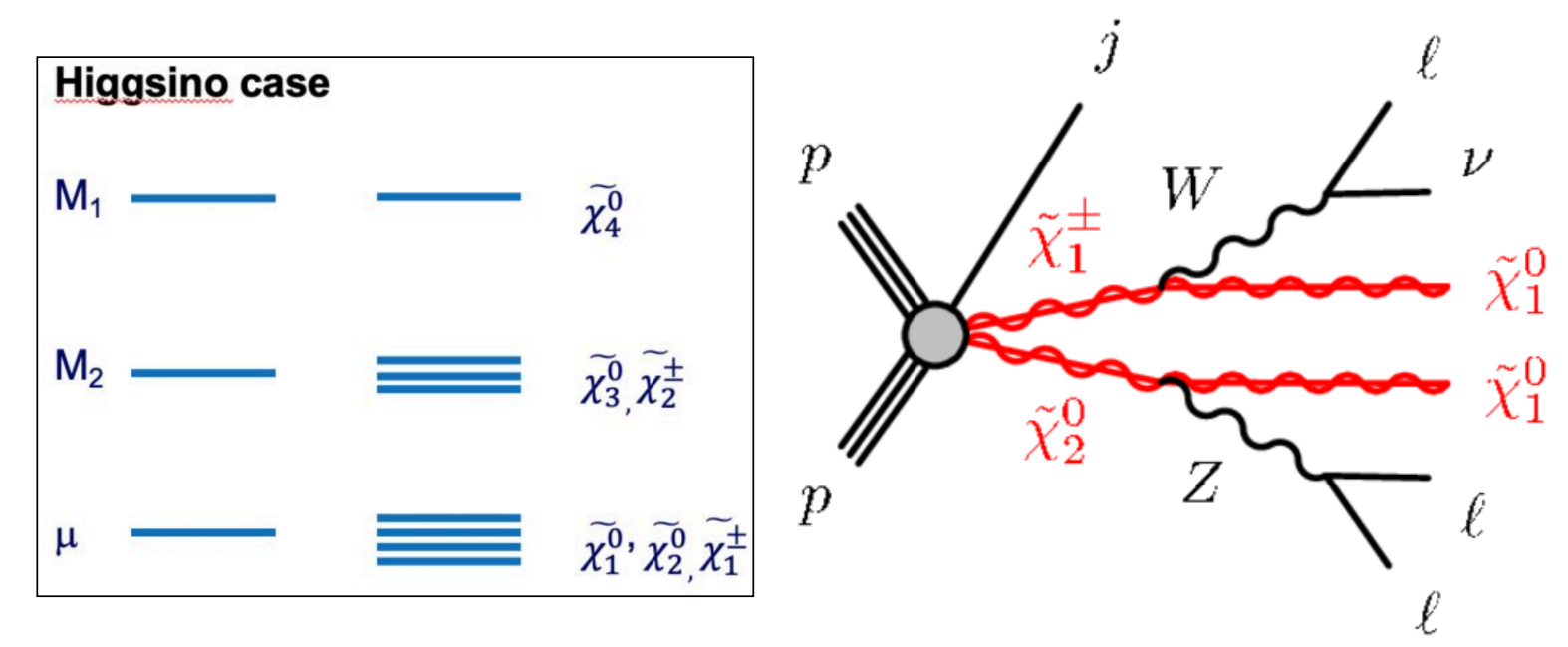

\begin{tabular}{|l|cc||c}
\hline Variable & $2 \ell$ Ewk & $3 \ell$ Ewk \\
& Low Med/High/Ultra & Low Med/High/Ultra \\
\hline \hline
\end{tabular}

$N_{\text {lep }} p_{\mathrm{T}}\left(\ell_{1}\right)[\mathrm{GeV}]$ for $\mu(\mathrm{e})$

$p_{\mathrm{T}}\left(\ell_{2}\right)[\mathrm{GeV}]$ for $\mu(\mathrm{e})$

$p_{\mathrm{T}}\left(\ell_{2}\right)[\mathrm{GeV}]$ for $\mu(\mathrm{e})$
$p_{\mathrm{T}}\left(\ell_{3}\right)[\mathrm{GeV}]$ for $\mu(\mathrm{e})$

1 OS pair

1 OSSF pair

$\mathrm{M}(\ell \ell)_{S F O S}\left(\mathrm{M}(\ell \ell)_{S F O S}^{\min }\right.$ in $\left.3 \ell\right)[\mathrm{GeV}$

$\mathrm{M}(\ell \ell)_{S F O S}\left(\mathrm{M}(\ell \ell)_{S F O S}^{\text {min }}\right.$ in $\left.3 \ell\right)[\mathrm{GeV}$

$\mathrm{M}(\ell \ell)_{S F O S}\left(\mathrm{M}(\ell \ell)_{S F O S}^{\text {min }}\right.$ in $\left.3 \ell\right)[\mathrm{GeV}$ $\mathrm{M}(\ell \ell)_{S F A S}^{\max }(\mathrm{AS}=$ any sign $)[\mathrm{GeV}]$ $p_{\mathrm{T}}(\ell \ell)[\mathrm{GeV}]$

$M_{T}\left(\ell_{i}, p_{\mathrm{T}}^{\text {miss }}\right)[\mathrm{GeV}](i=1,2)$

$\Delta R(\ell \ell)_{i j}(\mathrm{i}, \mathrm{j}=1,2,3)$

$p_{\mathrm{T}}^{\text {miss }}[\mathrm{GeV}]$

$p_{\mathrm{T}}^{\text {miss,corr }}[\mathrm{GeV}]$

$H_{\mathrm{T}}[\mathrm{GeV}]$

$p_{\mathrm{T}}^{\text {miss }} / H_{\mathrm{T}}$

Jet ID tight WP for leading jet

$\mathrm{N}_{b}\left(p_{\mathrm{T}}>25 \mathrm{GeV}\right)$

$M_{\tau \tau}$

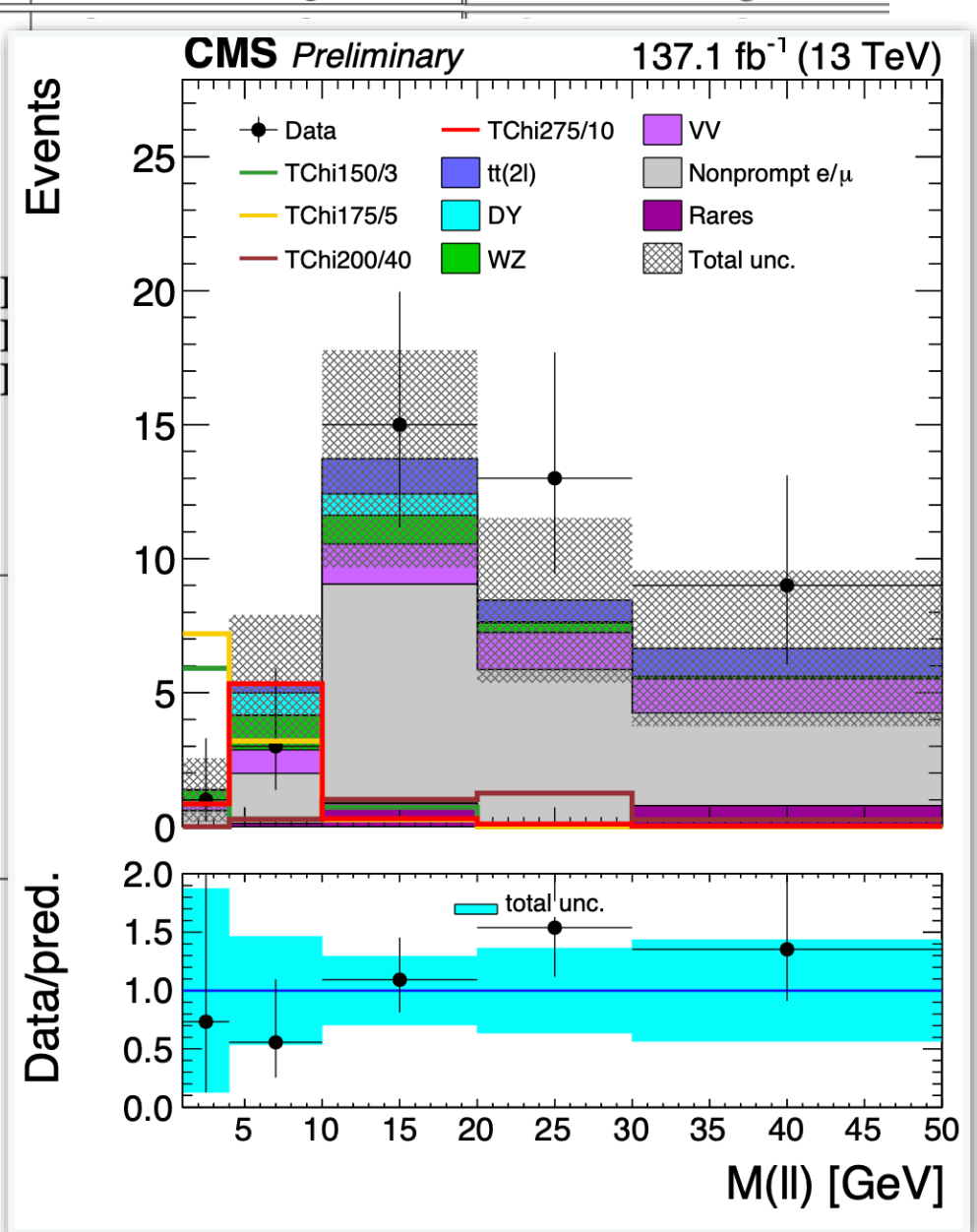

- Higgsino-like EWK-inos in the final state with one jet, moderate ETmiss and 2/3 soft leptons

- Muon (electron) pT > 3.5 (5) GeV

- Dilepton invariant mass down to $1 \mathrm{GeV}$

- Events classified based on ETmiss

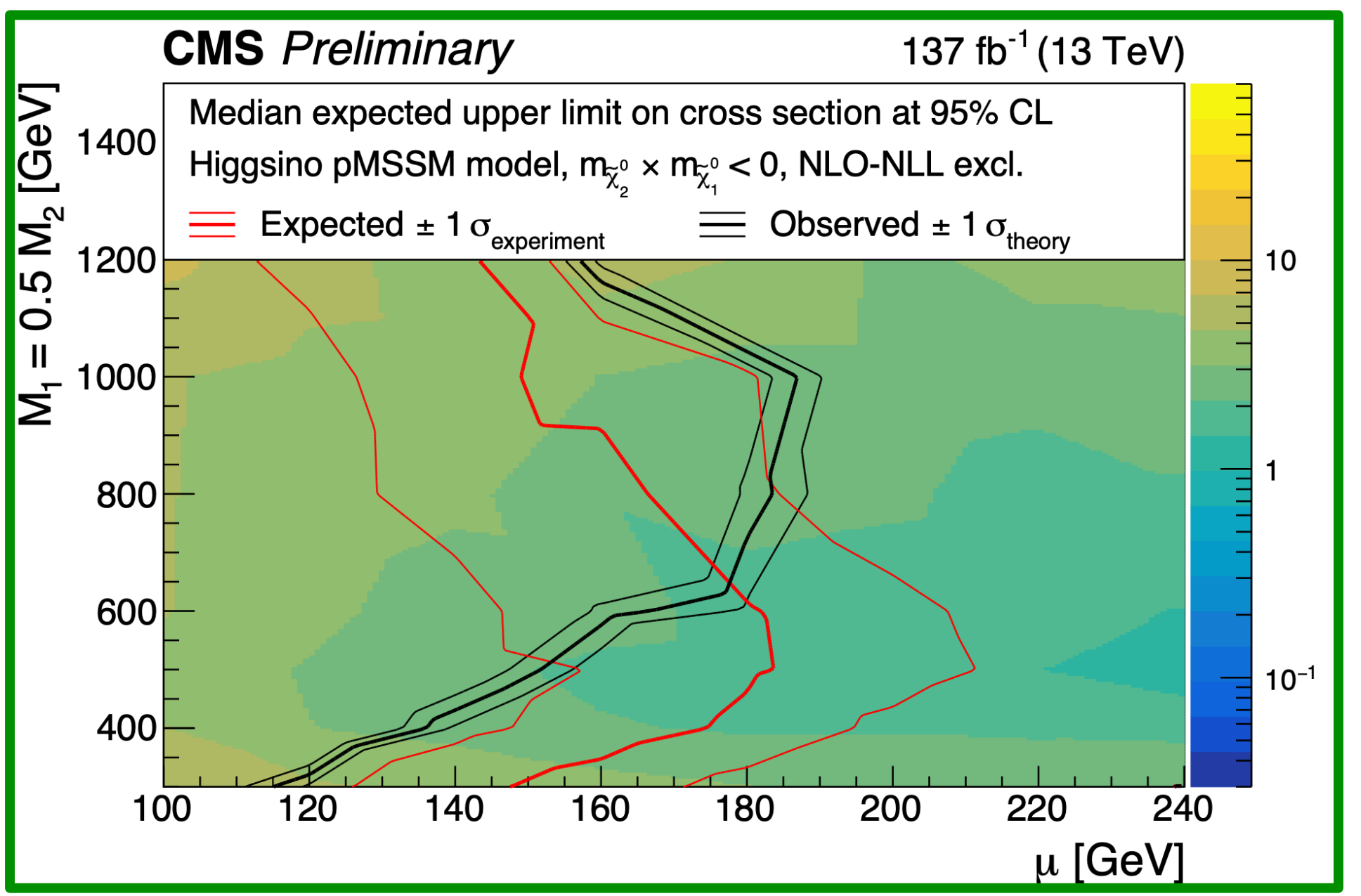

CMS-PAS-SUS-18-004 


\section{Search for long-lived charginos}

- Charginos decaying as $\tilde{\chi}^{ \pm} \rightarrow \pi^{ \pm} \chi^{0}$ expected in models with very compressed spectra

- Striking signature with a 'disappearing track'

- Pixel tracklets with no hits in the outermost silicon trackers and no calorimeter activity (newly introduced requirement)

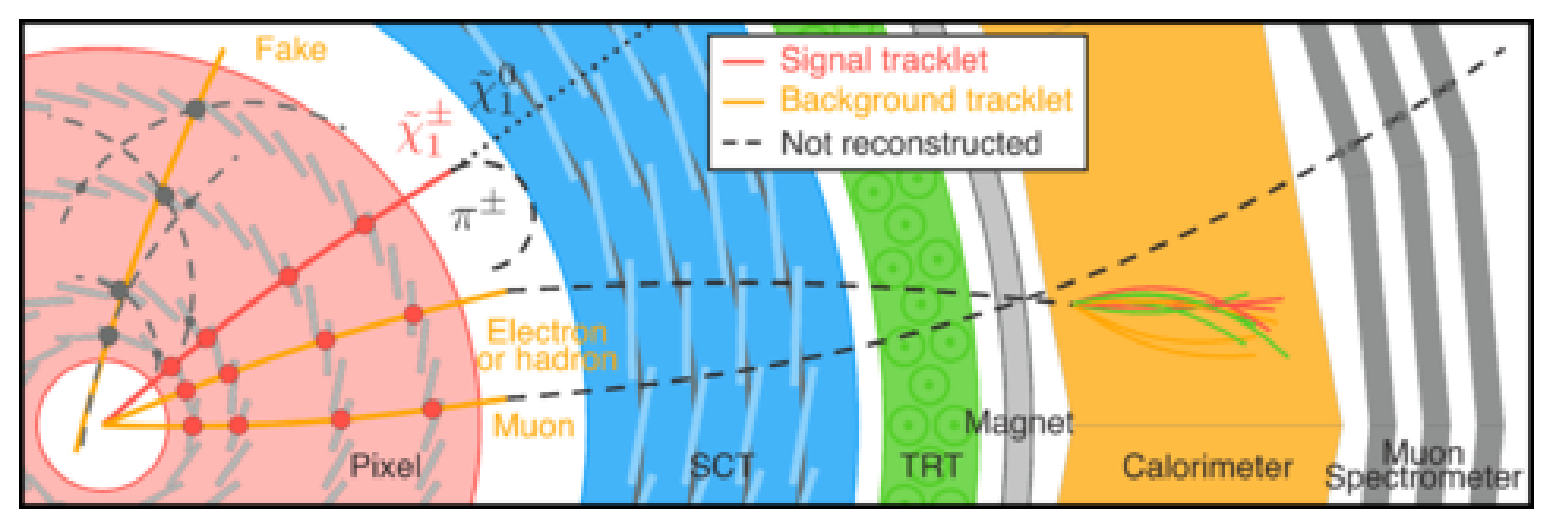

- ETmiss and number of jets for $\mathrm{S}$ to $\mathrm{B}$ discrimination

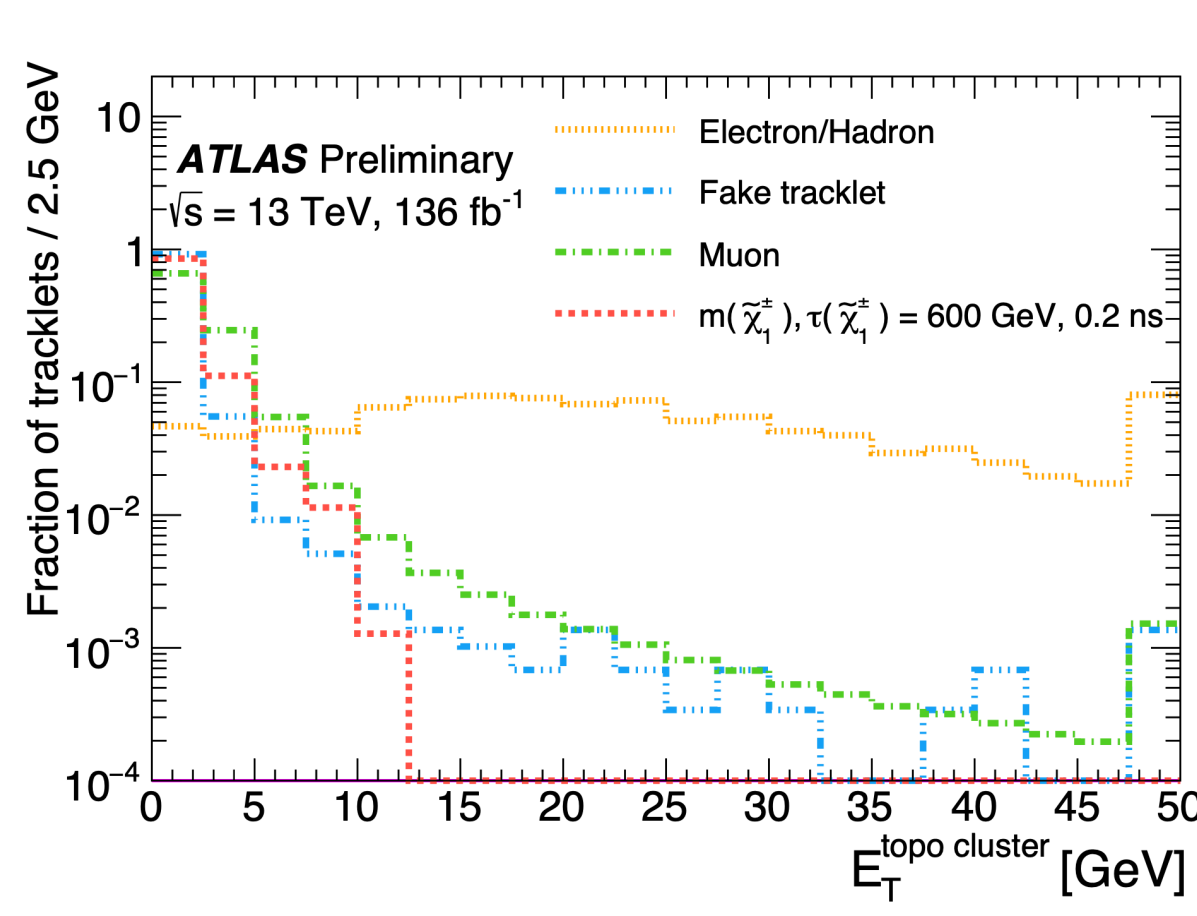

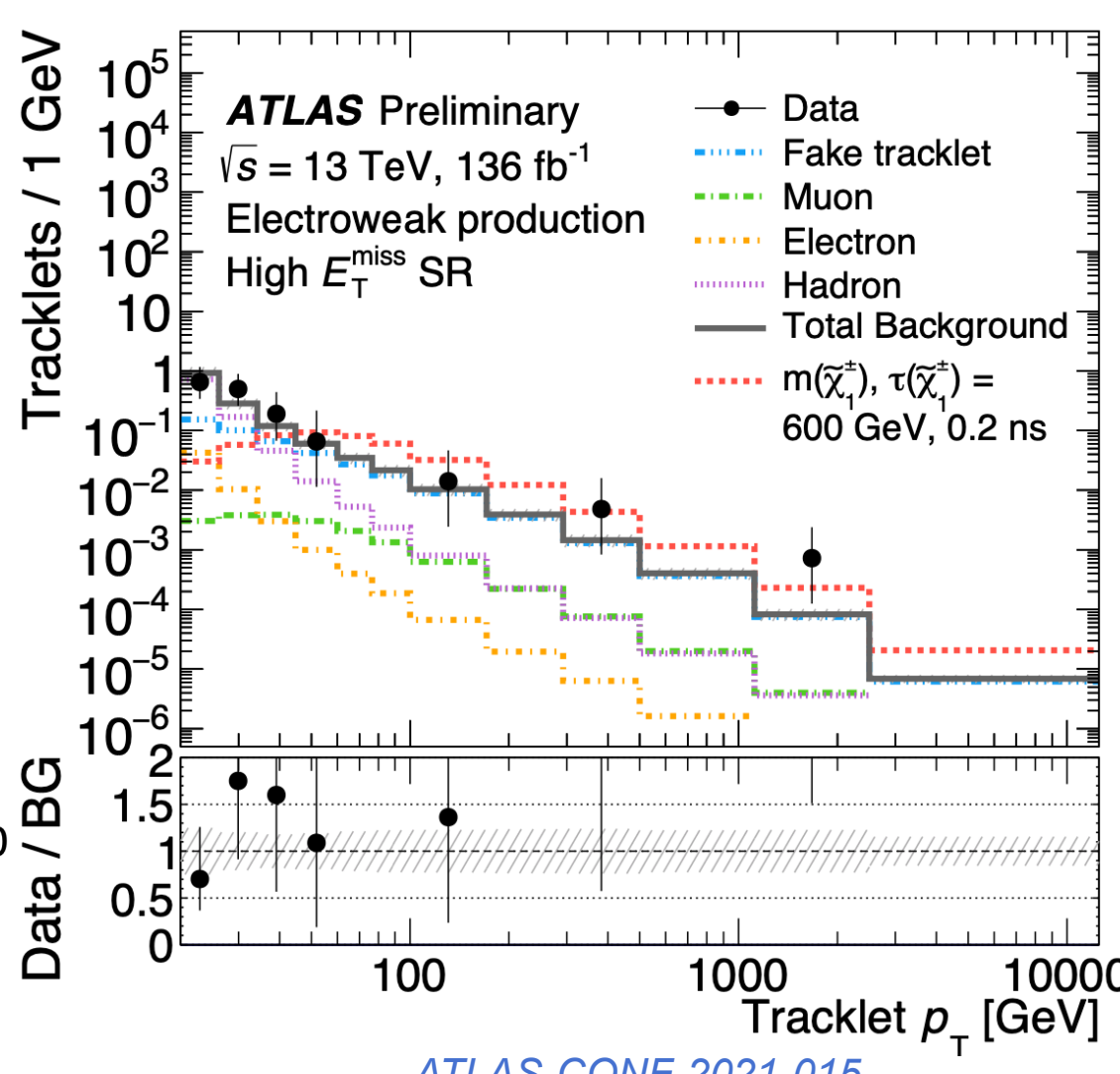

ATLAS-CONF-2021-015

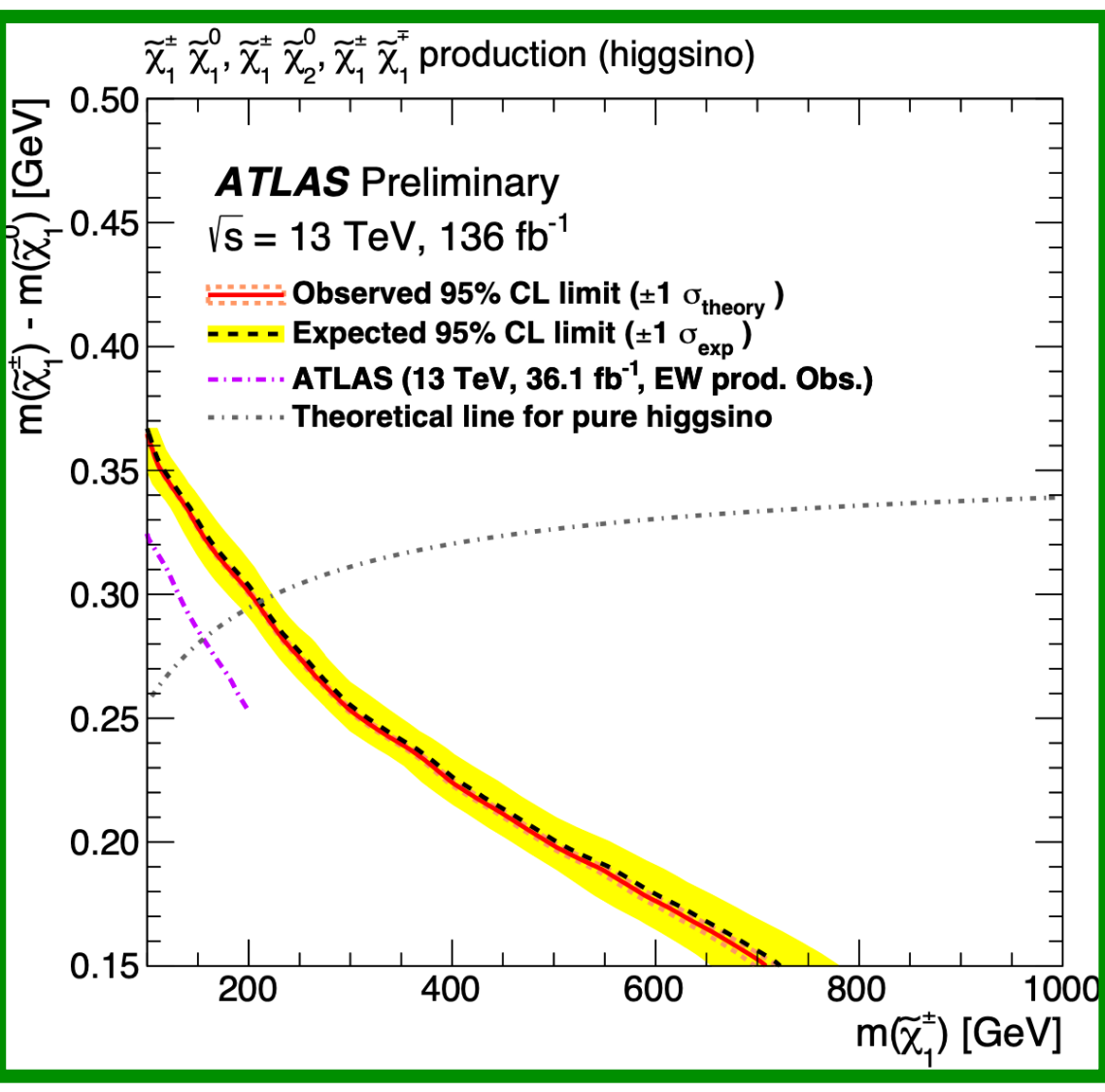

军 Fermilab 


\section{RPV SUSY in events with multiple (b-)jets}

- If $\lambda^{\prime \prime}$ dominant (as predicted under the minimal flavour violation hypothesis), decays into multi (b-) jets states

First LHC search

of this kind

- Search in events with one or two SS leptons, at least 8-15 (b-)jets sensitive to the production of gluinos, stops, electroweakinos

- Novel $b$-tagging algorithm, based on likelihood ratio of low-level tagger outputs, with adding RNNIP

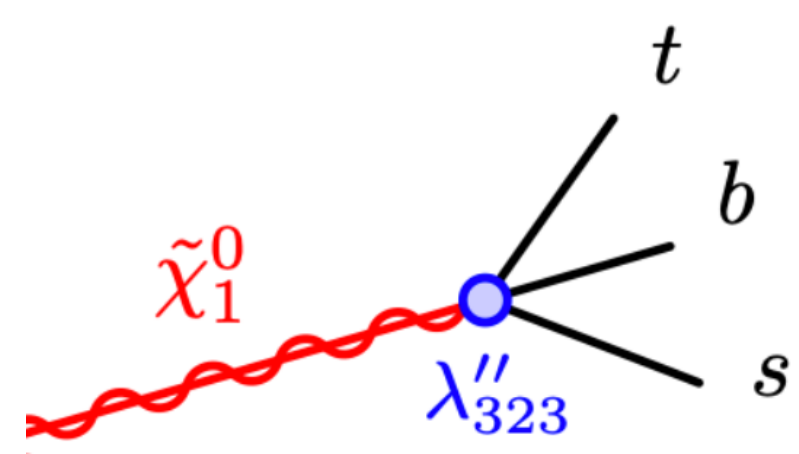

- Multivariate discriminants for EWK signal extraction

$D_{\mathrm{DL} 1}=\ln \left(\frac{p_{b}}{f_{c} \cdot p_{c}+\left(1-f_{c}\right) \cdot p_{\text {light }}}\right)$
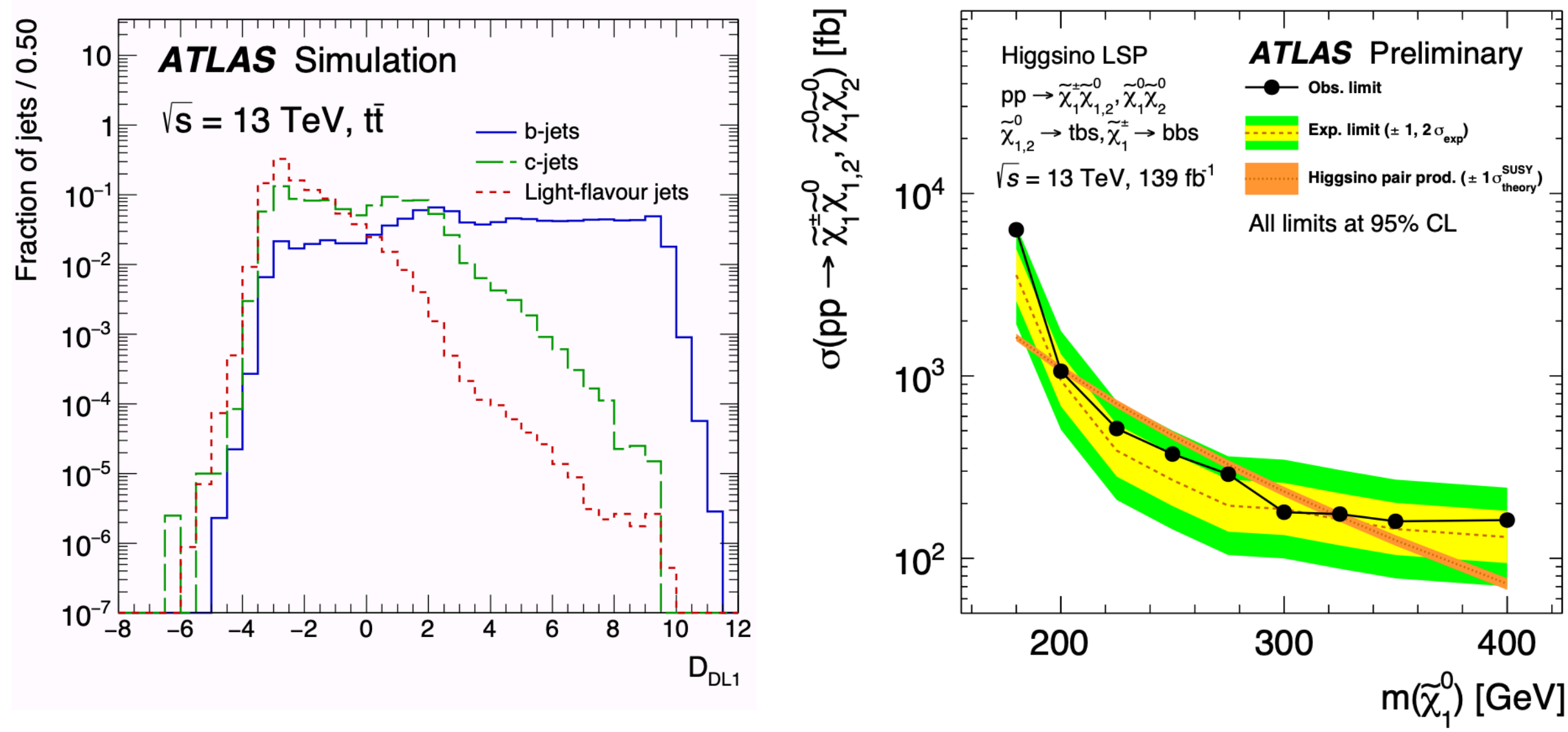


\section{New trigger opportunities at Run 3}

- At CMS

- global Level-1 triggers, for e.g. VBF signals

- low mass dimuon triggers using invariant mass

- triggers on single LLP leg

- increased data storage (data parking, data scouting)

- overall improved performances thanks to adoption of GPUs for tracking at high level trigger (allows to run particle flow)

- new triggers for long lived using upgraded calorimeter (better segmentation)

- $\ldots$ 


\section{New trigger opportunities at Run 3}

- At ATLAS

- global Level-1 triggers, for e.g. VBF signals

- Increased granularity of calorimeter information used in Level-1 triggers for electrons and photons

- More sophisticated Level-1 triggers for Taus, ETmiss (e.g. capability to trigger on large cone jets) and event-level quantities

- ML in tracking at High Level Trigger

- Increased data storage (data scouting, data parking)

- ... 